\title{
Norois
}

Environnement, aménagement, société

\section{Étude micromorphologique du Chemin Saulnier : apport à l'archéologie des paysages de la Plaine de Caen (Calvados, Basse-Normandie)}

Micromorphological study of the Chemin Saulnier: contribution to Landscape

archaeology of the Plain of Caen (Calvados, Normandy)

Irène Béguier, Cécile Germain-Vallée et Vincent Hincker

\section{OpenEdition}

\section{Journals}

Édition électronique

URL : http://journals.openedition.org/norois/3693

DOI : 10.4000 /norois.3693

ISSN : $1760-8546$

Éditeur

Presses universitaires de Rennes

Édition imprimée

Date de publication : 30 novembre 2011

Pagination : 121-142

ISBN : 978-2-7535-1765-3

ISSN : 0029-182X

\section{Référence électronique}

Irène Béguier, Cécile Germain-Vallée et Vincent Hincker, «Étude micromorphologique du Chemin Saulnier : apport à l'archéologie des paysages de la Plaine de Caen (Calvados, Basse-Normandie) », Norois [En ligne], 220 | 2011, mis en ligne le 30 novembre 2013, consulté le 19 avril 2019. URL : http:// journals.openedition.org/norois/3693 ; DOI : 10.4000/norois.3693

Ce document a été généré automatiquement le 19 avril 2019

(c) Tous droits réservés 


\title{
Étude micromorphologique du Chemin Saulnier : apport à l'archéologie des paysages de la Plaine de Caen (Calvados, Basse- Normandie)
}

\author{
Micromorphological study of the Chemin Saulnier: contribution to Landscape \\ archaeology of the Plain of Caen (Calvados, Normandy)
}

Irène Béguier, Cécile Germain-Vallée et Vincent Hincker

1 La question de la datation des cheminements anciens qui traversent la Plaine de Caen suscite de nombreuses réflexions. Certaines ont privilégié des datations antiques ou médiévales (Bertin, 1975; Doranlo, 1936-1937) et plus récemment des recherches archéogéographiques les font remonter à la Protohistoire, soit à l'Âge du Fer, soit à l'Âge du Bronze (Chevalier, 2009 ; Desloges, à paraître). C'est dans la perspective d'apporter de nouveaux éléments de datation sur ces axes anciens, qu'une étude géoarchéologique d'un chemin ancien appelé le Chemin Saulnier a été menée à l'occasion d'une opération de fouille préventive. Celle-ci a été réalisée dans les communes de Cagny et de Banneville-laCampagne au cours du printemps 2009 (Hincker et al., 2009). En croisant les approches archéologiques, géomorphologiques et paléopédologiques, cette recherche a permis d'analyser la genèse et l'évolution de cet axe de circulation ancien en relation avec des vestiges archéologiques le jouxtant, datés de la Protohistoire, de l'Antiquité et du Haut Moyen Âge. Outre ces derniers, c'est un tronçon du chemin lui-même qui a été étudié sur plusieurs centaines de mètres.

2 Son tracé, qui suit un axe rectiligne nord-sud entre les vallées de la Dives et de l'Orne (fig. 1), indique que le Chemin Saulnier devait avoir un rôle déterminant pour assurer le transit des hommes et des produits depuis l'estuaire de l'Orne en direction du sud de la Plaine de Caen. Cette dernière correspond à de vastes plateaux calcaires au relief 
faiblement ondulé, considérés comme un couloir de terres fertiles largement emprunté et habité depuis la Préhistoire comme en témoignent les recherches archéologiques et les prospections aériennes conduites dans ce secteur depuis une vingtaine d'années (Delacampagne et al., 1992 ; Desloges, 2001, 2002, à paraître ; Desloges et al., 2003, 2004).

La plus ancienne mention connue du Chemin Saulnier n'est pas antérieure à la fin $\mathrm{du}_{\mathrm{XII}}^{\mathrm{e}}$ siècle (Musset, 1983), mais les liens topographiques qu'il entretient en divers points avec des vestiges antiques ont depuis longtemps déjà fait supposer une origine plus ancienne. Parmi ces indices, il est possible de mentionner son passage au niveau de l'hypothétique agglomération secondaire antique de Bréville-les-Monts (Duvernois, 1987 ; Flotté, 2005) et son raccordement avec le tracé supposé de la voie reliant les villes antiques d'Augustodurum (Bayeux) et de Noviomagus (Lisieux) (fig. 2). Son appellation semble témoigner, par ailleurs, de son implication dans le commerce du sel, en lien avec les activités salicoles attestées dans l'estuaire de la Dives et qui semblent avoir eu des prolongements dans celui de l'Orne jusqu'aux environs de Sallenelles (Carpentier et al., 2006). Seule une étude archéologique directe de ce chemin, consistant à en fouiller un tronçon, permettait d'aborder la question de son origine. C'est dans cet objectif qu'a été engagée la fouille qui a donné lieu à la présente étude. Parallèlement, il a été possible de mettre en évidence, sous ce chemin, la présence de pédo-sédiments anciens. Ceux-ci ont été envisagés comme des archives sédimentaires (Fedoroff et Courty, 2002 ; Berger, 2006). Leur étude, comparée avec des observations faites ailleurs dans la Plaine de Caen, a permis de retracer l'évolution des paysages de ce secteur de la Plaine de Caen, au cours de l'Holocène, en reconstituant la succession des événements sédimentaires et pédologiques responsables de leur mise en place et de leur transformation post-dépositionnelle.

\section{Présentation de la zone d'étude}

\section{Le contexte géologique et géographique}

Si depuis l'Époque Moderne le Chemin Saulnier semble avoir perdu de son importance, comme en témoigne son absence sur la carte de Cassini, il n'en demeure pas moins encore nettement perceptible dans le paysage actuel sous la forme de tronçons de chemins ruraux, pour la plupart de terre. Son tracé peut encore être suivi sur une quinzaine de kilomètres depuis l'estuaire de l'Orne, jusqu'à son croisement avec l'ancienne voie reliant Vieux à Lisieux (fig. 2).

5 Le tronçon étudié est localisé dans la Plaine de Caen, à treize kilomètres en retrait du littoral de la Manche, sur un interfluve situé à la frontière des bassins versants de la basse vallée de l'Orne et de la Dives, à une altitude comprise entre 20 et 23 mètres NGF (fig. 1). Comme dans l'ensemble de la Plaine de Caen, ce plateau est caractérisé par un paysage d'openfield voué à la céréaliculture. Au nord-est, il est bordé par le ruisseau de Banneville et au sud par le ruisseau de Cagny. Orientés ouest-est, ces deux cours d'eau se jettent dans le ruisseau du Pont-de-Bâle, affluent de la Dives, qui alimente sur son passage le marais de Vimont situé à environ trois kilomètres au sud-est de la fenêtre d'étude (fig. 1). À l'ouest, un vallon sec orienté sud-nord délimite cet interfluve. Il rejoint la vallée de la rivière la Gronde, orientée est-ouest, qui conflue dans l'Orne à environ cinq kilomètres à l'ouest de la zone fouillée. Enfin, au nord, ce plateau s'incline doucement jusqu'à l'estuaire de l'Orne. 
Figure 1 : Localisation du Chemin Saulnier dans la moitié nord de la Plaine de Caen/Localization of the "Chemin Saulnier" in the northern half of the Plain of Caen

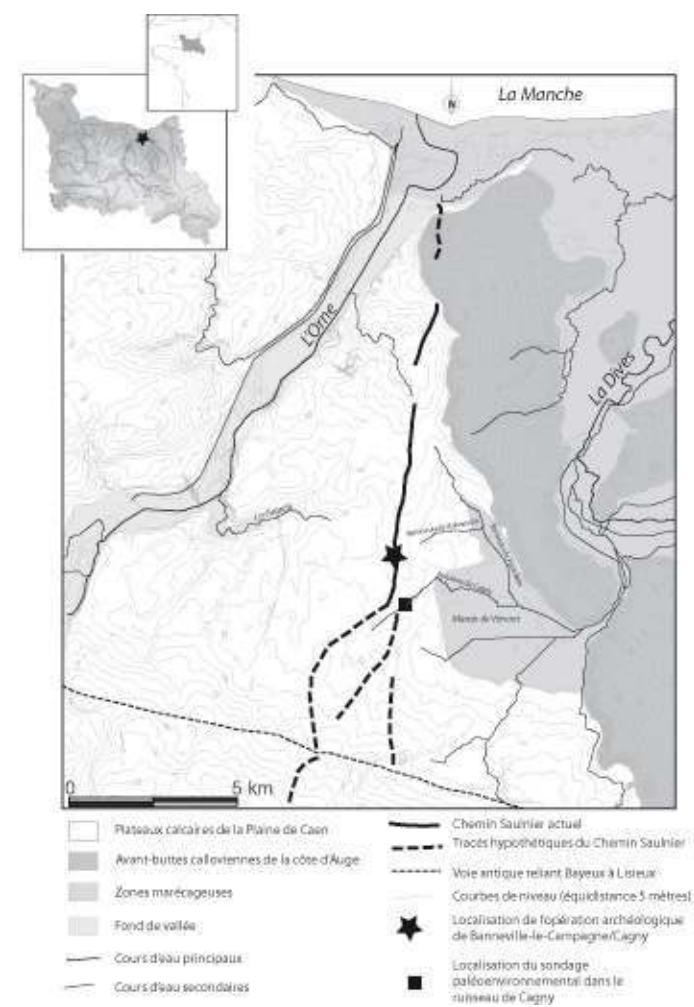

Figure 2 : Le Chemin Saulnier dans le réseau routier antique du Calvados central/The "Chemin Saulnier" in the central Calvados Roman road network

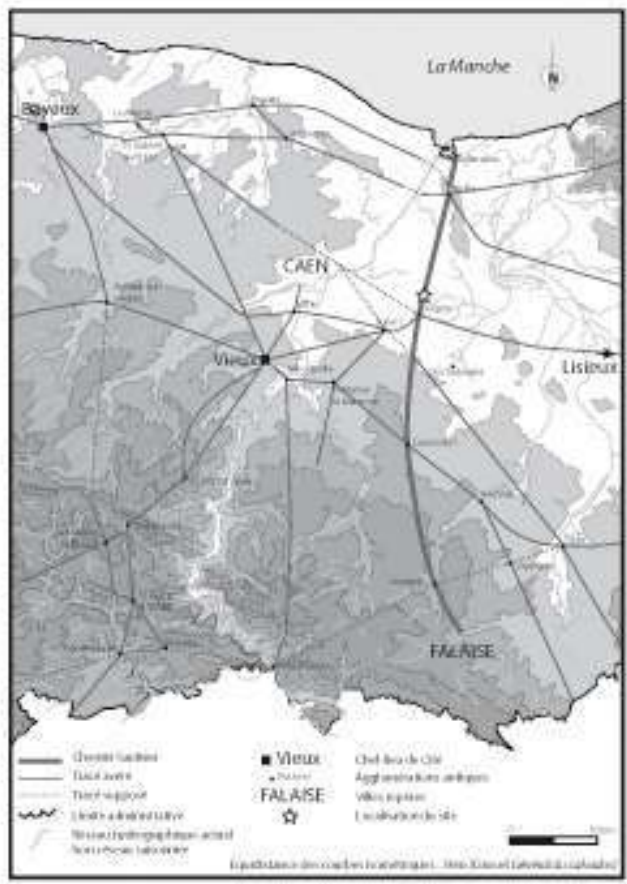

6 Le sous-sol est composé de marnes et de calcaires du Bathonien supérieur. Ces derniers sont surmontés d'une couverture loessique discontinue datée du Weichsélien (Coutard et 
al., 1970). Après une phase de décarbonatation survenue au Tardiglaciaire (Van VlietLanoë, 1988; Camuzard, 2000), des luvisols se sont développés, à partir du début de l'Holocène, aux dépens des loess, sous une couverture végétale protectrice de type chênaie-mixte atlantique (Fedoroff et Courty, 1994). Ces sols se définissent ordinairement par trois horizons pédologiques qui se sont différenciés au fur et à mesure des processus pédologiques de lessivage des particules argileuses depuis le sommet des horizons forestiers (Duchaufour, 1983). Ces trois horizons sont constitués du haut vers le bas : d'un horizon organique (A), d'un horizon éluvial (E) appauvri en argile et d'un horizon illuvial (Bt) enrichi en particules argileuses. Les mises en culture successives depuis les périodes anciennes ont provoqué généralement la dégradation puis l'ablation des horizons supérieurs $\mathrm{A}$ et $\mathrm{E}$ ainsi que la troncature partielle de l'horizon illuvial (Besnard-Vauterin et al., 2006 ; Camuzard, 2000 ; Germain-Vallée et Lespez, ce volume).

Dans la partie septentrionale de la zone fouillée, la couverture de loess présente une épaisseur variant entre 0,70 et $1,50 \mathrm{~m}$. Sa partie supérieure pédogénéisée se caractérise par un horizon limono-argileux brun rouge. Dans la partie méridionale, son épaisseur est moindre (de 0,20 à $0,40 \mathrm{~m}$ ) et le loess contient des graviers calcaires altérés ainsi que des restes macro-fossiles (brachiopodes, bivalves) issus du substratum marno-calcaire et calcaire sous-jacent. La formation pédologique qui s'est développée à ses dépens correspond à un horizon limono-argileux brun orangé surmonté d'un niveau de labour.

\section{Le contexte archéologique}

Dans la zone fouillée, de part et d'autre de l'autoroute A13, le Chemin Saulnier a pu être étudié sur une longueur de $420 \mathrm{~m}$. Il se présente sous la forme d'une chaussée large de 3 à $3,80 \mathrm{~m}$ et dont le tracé, assez linéaire, présente du nord au sud, un dénivelé d'1,80 m. Dans la partie méridionale de la zone fouillée, il est recouvert d'herbe rase et son profil en dôme surplombe d'un mètre les champs qui le bordent à l'ouest. À l'est, il est longé d'une haie boisée. Au nord de l'autoroute, il présente un profil plat d'une même altitude que les champs qui le jouxtent et est recouvert d'une couverture herbeuse plus clairsemée, absente des ornières. La fouille a permis d'identifier quatre états superposés de chemin sous la chaussée actuelle, alternativement en terre et en pierre. Cette évolution ne sera pas traitée plus avant dans cette contribution, dans la mesure où son objectif reste l'analyse de l'état le plus ancien. De part et d'autre de la chaussée actuelle, les vestiges archéologiques mis au jour révèlent trois phases d'occupation protohistoriques matérialisées par un ensemble d'enclos d'habitats fossoyés qui se succèdent entre la fin du Premier Âge du Fer et la fin de La Tène ancienne (Hincker et al., 2009) (fig. 3). À l'ouest $\mathrm{du}$ chemin, un enclos elliptique primitif se voit adjoindre un second enclos quadrangulaire, alors qu'au même moment un second enclos quadrangulaire de plus petite taille est installé de l'autre côté du chemin. Dans un troisième temps, les établissements fossoyés font place à un système de fossés parcellaires dont la mise en place intervient dans le courant du Second Âge du Fer. Au début de l'ère chrétienne, le parcellaire est à nouveau profondément remanié et le chemin est restructuré par la mise en place de la première chaussée empierrée (Hincker et al., 2009). 
Figure 3 : Plan synthétique du Chemin Saulnier et des enclos protohistoriques de l'opération de fouille préventive de Banneville-la-Campagne/Cagny (d'après Hincker et al., 2009)/Synthetic plan of the "Chemin Saulnier" and the Iron Age enclosure, the Banneville-la-Campagne/Cagny developer funded excavation (d'après Hincker et al., 2009)

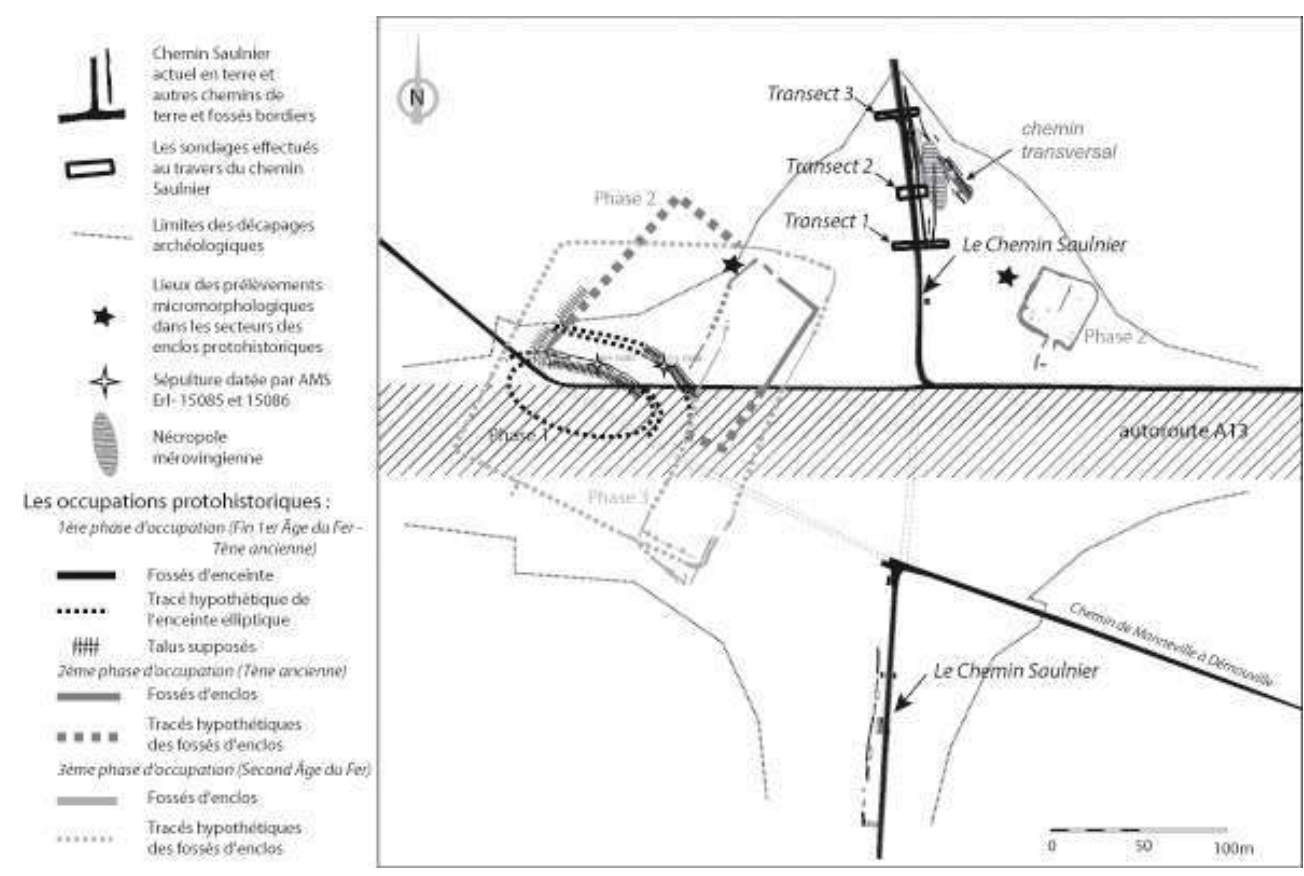

De très nombreux enclos de même nature ont été repérés dans les environs proches lors de diverses campagnes de prospection aérienne et attestent d'une forte densité d'occupation dans ce secteur de la Plaine de Caen au cours de la Protohistoire (Carte Archéologique, SRA Basse-Normandie) (fig. 4). Des indices d'installations antérieures, remontant au Néolithique, ont par ailleurs été collectés dans le voisinage immédiat de la zone fouillée à l'occasion de prospections de surface (Jasmin, 1942-1945). 
Figure 4 : Localisation des entités archéologiques d'époques pré- et proto-historiques repérées aux abords de la zone fouillée (d'après la Carte Archéologique - SRA Basse-Normandie)/Prehistoric, Bronze and Iron Age sites localized around the excavation zone (extract from the archaeological sites record - Service Régional de l'Archéologie, Lower-Normandy)

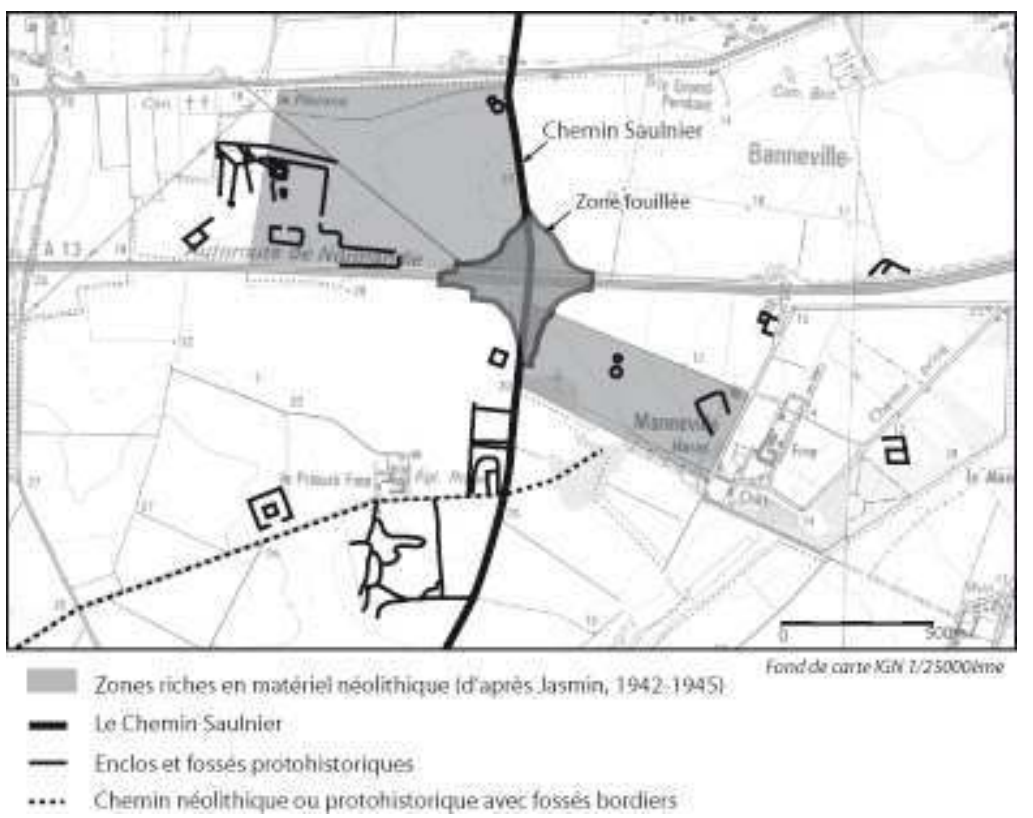

\section{Méthodologie}

L'étude géoarchéologique du Chemin Saulnier a été centrée sur le tronçon localisé dans la partie septentrionale de la zone fouillée qui présentait une stratigraphie plus développée, permettant d'envisager, dès la phase de terrain, un plus fort potentiel paléoenvironnemental. L'étude pédo-sédimentaire du tronçon situé au sud de l'autoroute a toutefois permis d'apporter des informations complémentaires pour la compréhension des formations pédologiques et sédimentaires de l'ensemble.

11 Au nord, le Chemin Saulnier a été analysé en procédant à l'ouverture de trois transects transversaux distants d'environ trente mètres, d'une longueur de vingt-quatre (transects 1 et 3) et quinze mètres (transect 2) (fig. 3). Les coupes stratigraphiques ainsi dégagées (coupes 1,2 et 3 ) ont fait l'objet d'un relevé détaillé à l'échelle $1 / 20$, suivi d'une description macroscopique de chacune des couches identifiées (texture, couleur, structure, distribution des éléments grossiers, nature des limites...). Dans un second temps, les décapages successifs des divers états du chemin ont permis de fouiller à plat les niveaux de circulation du chemin afin de collecter du mobilier archéologique susceptible de les dater (photo 1). Il s'agissait également d'étudier les relations stratigraphiques que ces niveaux entretenaient avec les autres structures archéologiques attenantes tels que les fossés bordiers. Par ailleurs, les décapages extensifs des enclos protohistoriques, situés à l'écart du secteur du Chemin Saulnier, ont permis l'observation détaillée et l'échantillonnage des pédo-sédiments du plateau. Comme pour les niveaux de circulation du chemin, la chronologie relative des divers épisodes pédo-sédimentaires rencontrés a été établie grâce à l'analyse typologique des mobiliers archéologiques qu'ils contenaient ou en se fondant sur la datation des vestiges archéologiques avec lesquels ils avaient un lien stratigraphique. Deux datations radiométriques (AMS) ont également été réalisées 
sur des ossements en provenance de deux sépultures associées aux deux enclos d'habitat protohistoriques les plus anciens situés à l'ouest du chemin (tableau 1, fig. 1). Enfin, deux datations radiométriques (AMS) complémentaires ont été effectuées sur des charbons prélevés dans la stratigraphie fossilisée par le chemin et dépourvue de mobilier archéologique (tableau 1, fig. 5).

Les observations de terrain ont été complétées par une étude micromorphologique. Dixhuit échantillons en blocs non perturbés ont été prélevés dans les trois coupes stratigraphiques du chemin et cinq dans les pédo-sédiments présents dans les secteurs à enclos protohistoriques, à l'écart du chemin. Ces prélèvements ont été effectués de préférence à la transition entre deux unités pédo-sédimentaires (fig.5). Après leur induration dans de la résine polyester, ils ont été montés en lames minces de grand format $(9 \times 16 \mathrm{~cm})$ puis analysés au microscope polarisant selon un système descriptif inspiré des manuels de référence en micromorphologie (Bullock et al., 1985 ; Courty et al., 1989, 1994 ; Fedoroff et Courty, 1994, 2002).

L'analyse des sédiments sous le microscope permet l'identification de traits pédologiques et sédimentaires. Les traits sédimentaires informent sur le mode de mise en place des sédiments, tandis que les traits pédologiques correspondent aux transformations qu'ils ont subies après leur dépôt sous l'action des agents atmosphériques, de la faune et de la flore. Dans les sédiments étudiés, les principaux traits pédologiques rencontrés sont des traits biologiques (porosité canaliculaire, organisations excrémentales, carbonatations secondaires en aiguilles, etc.), texturaux (liés à des concentrations d'argiles et de limons sous la forme de revêtements en bordure des vides ou d'intercalations dans la matrice du sol), carbonatés (hyporevêtements carbonatés) et ferrugineux témoignant d'une certaine hydromorphie (nodules, imprégnations ferrugineuses, etc.). L'importance de ces traits pédologiques permet, par exemple, de déduire des phases de stabilité paysagère qui, pour la période holocène, suggèrent des périodes de déprise agricole (Berger, 2006). Les interruptions dans la mise en valeur des sols entraînent, en effet, leur reconquête par un couvert végétal protecteur qui se manifeste par le développement de traits pédologiques caractéristiques d'une reprise de la pédogénèse. Enfin, l'analyse en lame mince a permis d'observer une dernière catégorie de traits d'origine anthropique, liés aux piétinements (Courty et al., 1989 ; Courty et Fedoroff, 2002).

Tableau 1 : Datation par méthode physico-chimique (AMS) à partir d'ossements et de microcharbons prélevés respectivement dans des sépultures et des pédo-sédiments, mis au jour sur le site de Banneville-la-Campagne/Cagny/Dating by a physico-chemical method (AMS) from bones and charcoal taken respectively from graves, and pedo-sediments from the Banneville-la-Campagne/ Cagny site

\begin{tabular}{|l|l|l|l|}
\hline $\begin{array}{l}\text { Référence } \\
\text { échantillon }\end{array}$ & $\begin{array}{l}\text { Nature de } \\
\text { l'échantillon }\end{array}$ & Datation par AMS (à 2 sigma) & Identification \\
\hline Erl - 15082 & Microcharbons & $\begin{array}{l}4182 \pm 36 \mathrm{BP} \\
\text { soit 2817-2661 av. J-C. cal. }\end{array}$ & $\begin{array}{l}\text { Horizon illuvial (Bt) } \\
\text { (coupe 1, niveau 2) }\end{array}$ \\
\hline Erl - 15083 & Microcharbons & $\begin{array}{l}3416 \pm 33 \mathrm{BP} \\
\text { soit 1776-1625 av. J.-C. cal. }\end{array}$ & $\begin{array}{l}\text { Paléosol } \\
\text { (coupe 1, niveau 3) }\end{array}$ \\
\hline Erl - 15084 & Ossements & $\begin{array}{l}2432 \pm 34 \mathrm{BP} \\
\text { soit 594-403 av. J.-C. cal. }\end{array}$ & Sépulture 5009A \\
\hline
\end{tabular}




\begin{tabular}{|l|l|l|l|}
\hline Erl - 15085 & Ossements & $\begin{array}{l}2436 \pm 36 \mathrm{BP} \\
\text { soit 595-404 av. J.-C. }\end{array}$ & Sépulture 5013 \\
\hline
\end{tabular}

L'ensemble des datations retenues pour la présente étude correspond à des âges calibrés avec 95,4 \% de probabilité (2 sigma). Les mesures ont été effectuées par AMS-Labor Erlangen, Autriche.

Photo 1 : Un niveau de circulation antique (II--III siècle apr. J.-C.) du Chemin Saulnier/An Roman level of circulation level ( $2^{\text {nd }}-3^{\text {rd }}$ centuries A.D.) of the Chemin Saulnier

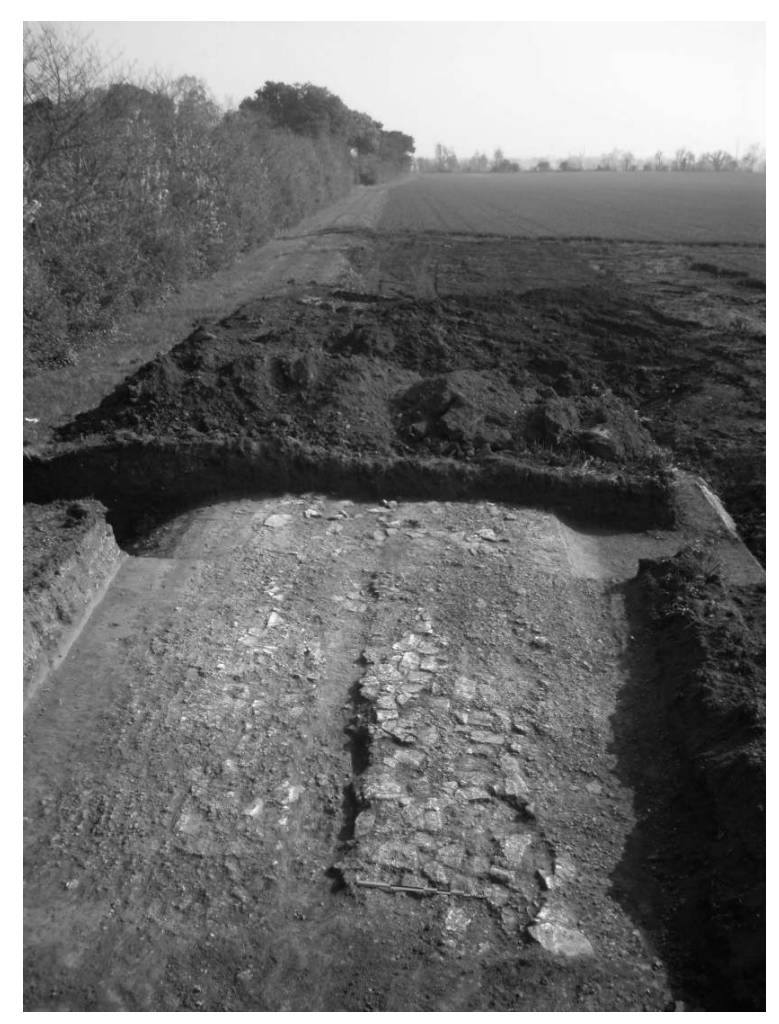

\section{Les formations pédo-sédimentaires de la zone étudiée}

14 Les trois coupes relevées de la partie septentrionale de la zone fouillée, montrent une stratigraphie similaire (présence des pédo-sédiments et des différents états du Chemin Saulnier). Toutefois, comme dans les coupes 2 et 3 le premier état du chemin est moins bien conservé, la coupe 1 (transect 1) a été choisie comme coupe de référence pour cet article (fig. 5). En se basant sur cette coupe et sur celles relevées dans les secteurs des enclos protohistoriques, il apparaît que la stratigraphie au nord de la zone fouillée comprend, au-dessus du substrat loessique, deux à cinq épisodes pédo-sédimentaires successifs. Ces séquences pédo-sédimentaires ont été schématisées dans la figure 6 . 
Figure 5 : La coupe 1 du Chemin Saulnier (transect 1)/Section 1 of the Chemin Saulnier

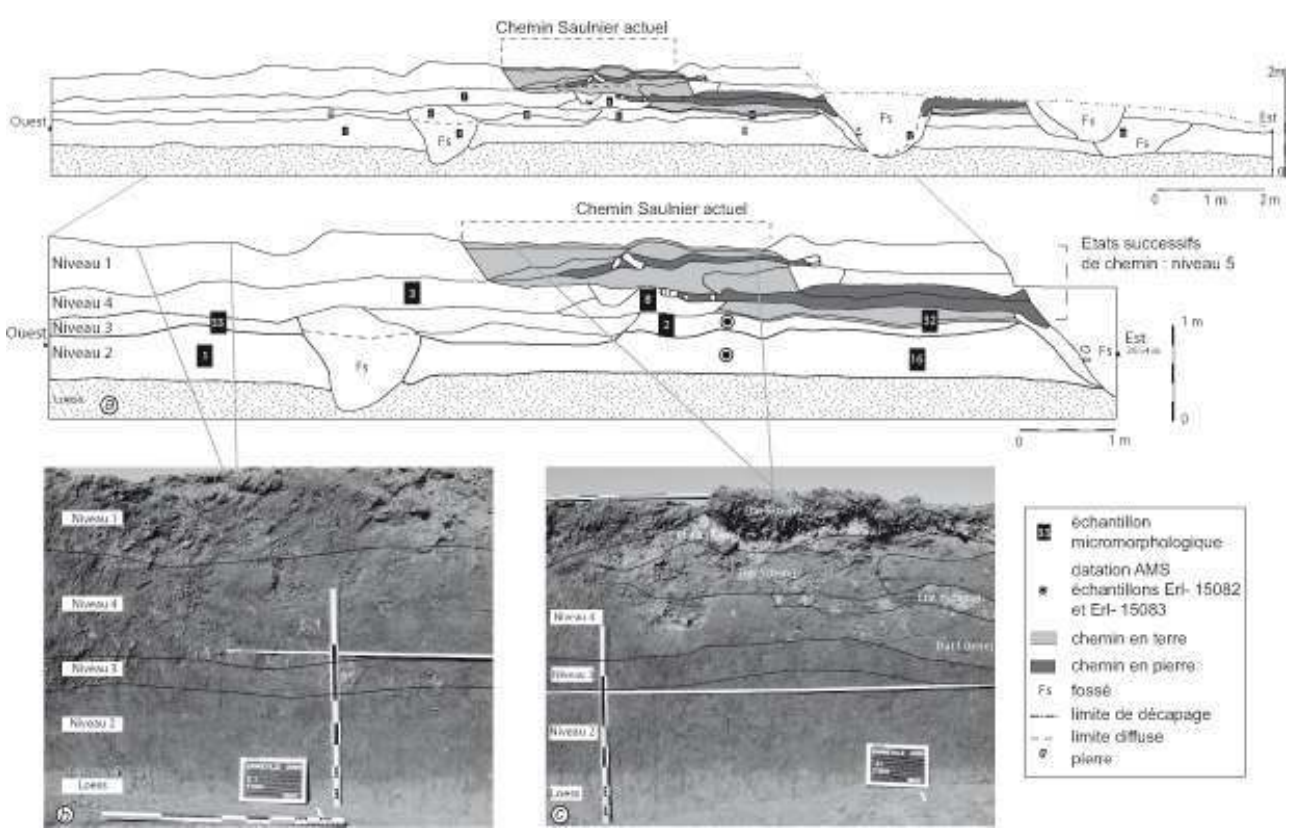

Figure 6 : Restitution des trois profils pédo-sédimentaires analysés sur le site de Banneville-laCampagne/Cagny/Reconstruction of three pedo-sedimentary profiles from the Banneville-la-Campagne/ Cagny archaeological site

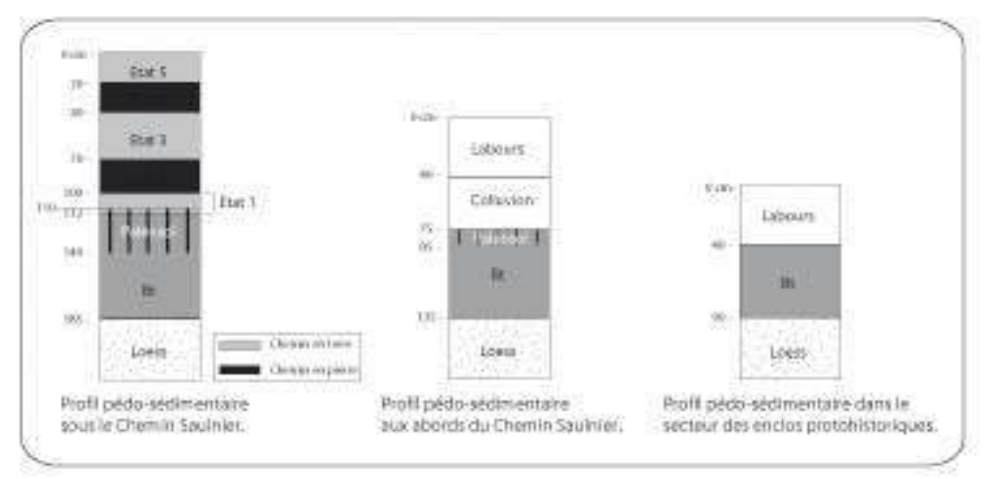

15 À l'est et à l'ouest du chemin, à hauteur des enclos protohistoriques, la séquence stratigraphique qui surmonte un niveau de loess, comprend un premier horizon à structure prismatique peu développée, brun-rouge limoneux à limono-argileux épais de $50 \mathrm{~cm}$ (niveau 2). Il est recouvert par un horizon limoneux brun-gris de $40 \mathrm{~cm}$ d'épaisseur qui correspond aux labours actuels (niveau 1). En bordure de la chaussée, entre ces deux horizons s'intercalent deux autres niveaux: un premier, brun sombre grumeleux de $10 \mathrm{~cm}$ d'épaisseur (niveau 3) recouvert par un second limono-graveleux brun, épais de $30 \mathrm{~cm}$ (niveau 4).

16 Au niveau du tracé actuel du chemin, l'horizon de labour est remplacé par une série de couches stratigraphiques composées soit de pierres et de cailloux fortement compactés soit de sédiments brun-jaune verdâtres limoneux caractérisés par une structure lamellaire très développée. L'ensemble de ces couches, qui se développent sur $1,12 \mathrm{~m}$ d'épaisseur et s'étirent sur une largeur de 3 à 7,50 m, signale la succession des différents niveaux de circulation du Chemin Saulnier. Ceux-ci sont regroupés arbitrairement en un 
seul événement stratigraphique (niveau 5). Sous ces derniers se retrouve la succession du loess, de l'horizon brun-rouge (niveau 2) et du niveau brun sombre grumeleux (niveau 3), identique à celle observée en bordure immédiate de la chaussée. Toujours sous ces différents états de chemin, le niveau brun sombre grumeleux (niveau 3) présente en surface, sur 2 à $4 \mathrm{~cm}$ d'épaisseur, une teinte plus sombre, brun-noir, ainsi que des indices importants de tassement tels qu'une structure lamellaire très développée. Ces indices tendent à montrer que ce niveau fait figure d'assise au premier état en terre du Chemin Saulnier.

17 Hormis l'horizon de labour, toutes ces couches sédimentaires ont fait l'objet d'une analyse micromorphologique. Les principaux traits pédologiques rencontrés pour chaque unité pédo-sédimentaire individualisée dans les différents lieux d'observation (dans les secteurs des enclos, aux abords du chemin et sous ce dernier) ont été reportés de manière synthétique dans les tableaux 2, 3 et 4 .

Tableau 2 : Description synthétique macroscopique et microscopique des niveaux observés dans le secteur des enclos protohistoriques/Synthetic macroscopic and microscopic description of the levels observed in the sector of the protohistoric enclosures

\begin{tabular}{|c|c|c|c|c|}
\hline $\begin{array}{l}\text { Stratigraphie } \\
\text { hors secteur } \\
\text { du chemin }\end{array}$ & $\begin{array}{l}\text { Observations } \\
\text { macroscopiques }\end{array}$ & $\begin{array}{l}\text { Observations } \\
\text { micromorphologiques }\end{array}$ & Datation & Interprétation \\
\hline Niveau 1 & $\begin{array}{l}\text { Niveau limono- } \\
\text { sableux brun-gris, } \\
\text { peu compact à } \\
\text { meuble, structure } \\
\text { granuleuse avec } \\
\text { quelques graviers } \\
\text { et pierres. }\end{array}$ & & $\begin{array}{l}\text { Époque } \\
\text { Moderne }\end{array}$ & $\begin{array}{l}\text { Horizon de } \\
\text { labour }\end{array}$ \\
\hline Niveau 2 & $\begin{array}{l}\text { Niveau limoneux à } \\
\text { limono-argileux } \\
\text { brun-rouge orangé } \\
(7,5 \text { et } 10 \text { YR } 5 / 6 \\
4 / 6), \text { compact, } \\
\text { structure } \\
\text { prismatique assez } \\
\text { développée, } \\
\text { quelques éléments } \\
\text { grossiers. }\end{array}$ & 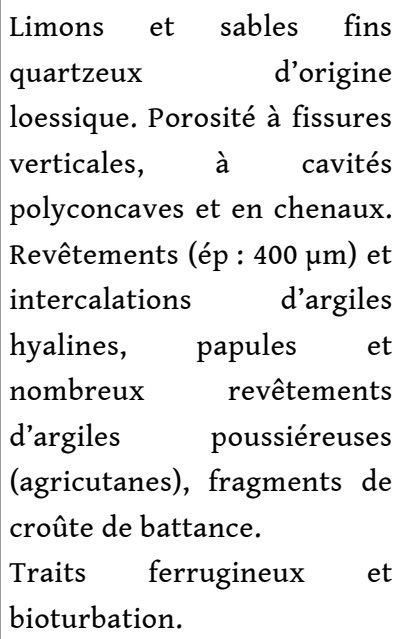 & & $\begin{array}{l}\text { Horizon illuvial } \\
(\mathrm{Bt}) \text { issu d'un } \\
\text { luvisol tronqué } \\
\text { dont la surface } \\
\text { est } \\
\text { régulièrement } \\
\text { mise à nue suite } \\
\text { à une activité } \\
\text { agricole } \\
\text { soutenue. }\end{array}$ \\
\hline
\end{tabular}




\section{Principaux traits micromorphologiques et interprétation des pédo- sédiments étudiés}

\section{Le niveau 2: L'horizon limono-argileux brun-rouge (tableaux 2, 3 et 4)}

18 À l'échelle microscopique, les nombreux revêtements bruns-jaunes à orangés d'argiles hyalines (photo 2), caractéristiques d'un horizon illuvial d'un luvisol, ne font aucun doute sur la nature de l'horizon limono-argileux brun-rouge surmontant les loess. Il s'agit de l'horizon Bt d'un luvisol tronqué. Ce constat s'accorde avec les observations faites partout sur les plateaux de la moitié nord de la Plaine de Caen où ne subsiste que l'horizon illuvial (Bt) de ces luvisols (Coutard et al., 1970 ; Besnard-Vauterin et al., 2006 ; Germain-Vallée et Lespez, ce volume).

Dans la zone d'étude, cet horizon Bt comporte de nombreux traits texturaux tels que des papules (photo 3), qui indiquent des phases alternées d'érosion et de colluvionnement ainsi que des revêtements d'argiles poussiéreuses (photo 4) qui témoignent d'un dénuement de la surface (Fedoroff et Courty, 2002 ; Weber-Tièche et Sordoillet, 2008). On note également la présence de nombreuses intercalations et de traits ferrugineux qui suggèrent des engorgements marqués (Fedoroff et Courty, 2002). Si l'horizon illuvial présente le même type d'organisation pédo-sédimentaire sous, aux abords et en dehors du chemin, il se distingue au niveau des enclos protohistoriques par la présence de fréquents fragments de croûtes de battance (photo 5) qui attestent de précipitations sur un sol mis à nu, certainement en relation avec une activité agricole soutenue (Fedoroff et Courty, 2002). Dans ce contexte sédimentaire, les revêtements d'argiles poussiéreuses observés peuvent être interprétés comme des agricutanes (Gebhardt, 1990; Fedoroff et Courty, 1994). En résumé, l'horizon limono-argileux brun-rouge qui surmonte le loess (niveau 2) témoigne d'une importante phase de pédogénèse qui a permis le développement d'un luvisol, qui a ensuite été tronqué. L'importante phase érosive qui a provoqué cette phase de troncature/colluvionnement de l'horizon illuvial, s'est déroulée avant l'installation du chemin.

Tableau 3 : Description synthétique macroscopique et microscopique des niveaux observés aux abords du Chemin Saulnier, d'après la coupe 1 (fig. 5)/Synthetic macroscopic and microscopic description of the levels observed around the Chemin Saulnier, according to the section 1 (fig. 5)

\begin{tabular}{|c|c|c|c|c|}
\hline $\mid \begin{array}{lr}\text { Stratigraphie } \\
\text { près } & \mathrm{du}\end{array}$ & $\begin{array}{l}\text { Observations } \\
\text { macroscopiques }\end{array}$ & $\begin{array}{l}\text { Observations } \\
\text { micromorphologiques }\end{array}$ & Datation & Interprétation \\
\hline Niveau 1 & $\begin{array}{l}\text { Niveau limono- } \\
\text { sableux brun- } \\
\text { gris, peu compact } \\
\text { à meuble, } \\
\text { structure } \\
\text { granuleuse avec } \\
\text { quelques graviers } \\
\text { et pierres. }\end{array}$ & & Moderne & $\begin{array}{l}\text { Horizon de } \\
\text { labour }\end{array}$ \\
\hline
\end{tabular}




\begin{tabular}{|c|c|c|c|c|}
\hline Niveau 4 & $\begin{array}{l}\text { Limon graveleux } \\
\text { brun foncé à } \\
\text { clair, peu } \\
\text { compact, } \\
\text { structure } \\
\text { grumeleuse, } \\
\text { graviers, cailloux } \\
\text { et pierres } \\
\text { calcaires. }\end{array}$ & $\begin{array}{l}\text { Limons et sables fins } \\
\text { quartzeux, } \\
\text { fragments de silex sub- } \\
\text { anguleux et de calcaire } \\
\text { bioclastique. Microstructure } \\
\text { massive à grumeleuse à } \\
\text { porosité en chenaux. } \\
\text { Nombreux revêtements } \\
\text { d'argiles poussiéreuses et } \\
\text { rares fragments d'argiles } \\
\text { hyalines. Rares aiguilles et } \\
\text { pseudomorphoses racinaires } \\
\text { calcifiées. Quelques traits } \\
\text { ferrugineux. Bioturbation } \\
\text { (chambre, organisation } \\
\text { excrémentale en pile } \\
\text { d'assiette). }\end{array}$ & $\begin{array}{l}\text { Période } \\
\text { antique }\end{array}$ & $\begin{array}{l}\text { Colluvion } \\
\text { (rideau de } \\
\text { culture) issue de } \\
\text { l'érosion des } \\
\text { paléosols. Le } \\
\text { développement } \\
\text { d'une végétation } \\
\text { clairsemée } \\
\text { atteste d'une } \\
\text { courte période } \\
\text { de stabilité. }\end{array}$ \\
\hline Niveau 3 & 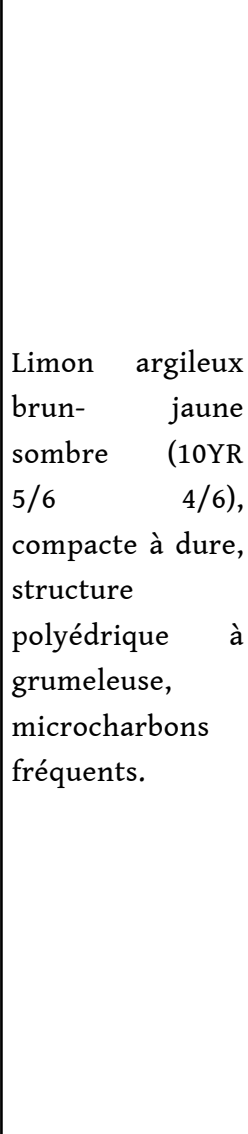 & 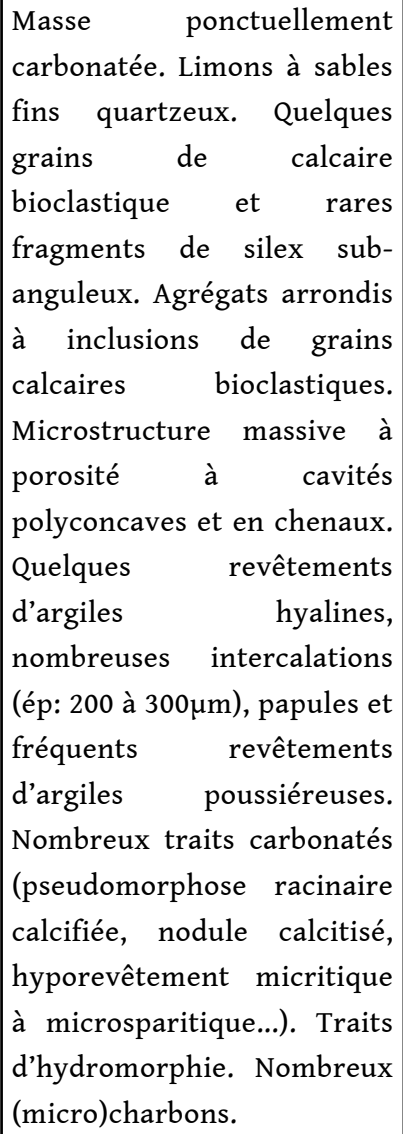 & 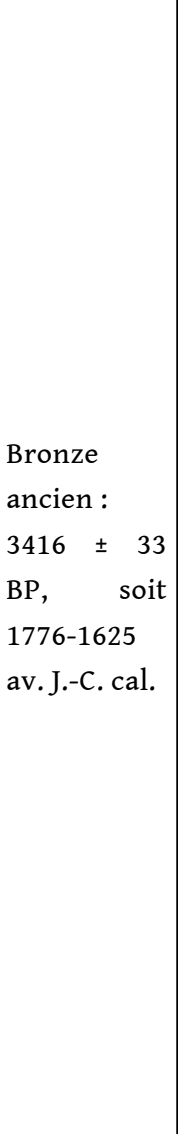 & $\begin{array}{l}\text { Paléosol } \\
\text { développé aux } \\
\text { dépens du Bt et } \\
\text { dont la } \\
\text { couverture } \\
\text { végétale a été } \\
\text { défrichée } \\
\text { (brûlis?). }\end{array}$ \\
\hline
\end{tabular}




\begin{tabular}{|c|c|c|c|c|}
\hline Niveau 2 & $\begin{array}{l}\text { Limon argileux } \\
\text { brun- rouge } \\
\text { orangé à jaune, } \\
\text { très compact, } \\
\text { structure } \\
\text { prismatique } \\
\text { assez développée, } \\
\text { microcharbons } \\
\text { épars, quelques } \\
\text { concrétions } \\
\text { carbonatées. }\end{array}$ & 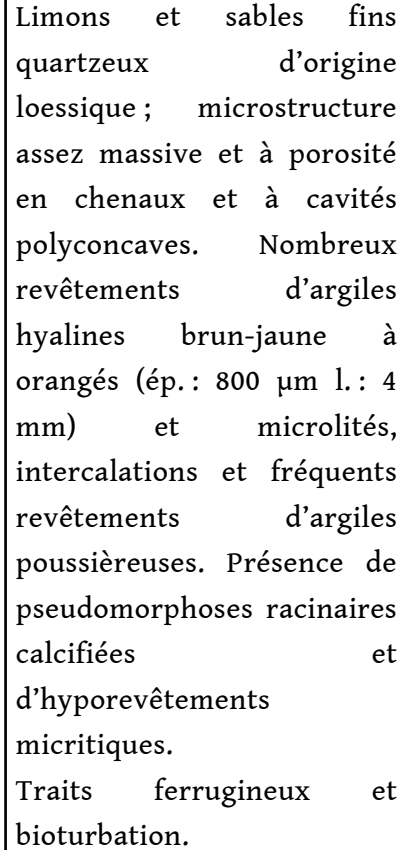 & $\begin{array}{l}\text { Néolithique } \\
\text { final : } 4182 \\
\pm 36 \text { BP, soit } \\
2817-2661 \\
\text { av. J.-C. cal. }\end{array}$ & $\begin{array}{l}\text { Horizon illuvial } \\
\text { (Bt) issu d'un } \\
\text { luvisol tronqué. }\end{array}$ \\
\hline
\end{tabular}

Tableau 4 : Description synthétique macroscopique et microscopique des niveaux observés sous le Chemin Saulnier, d'après la coupe 1 (fig. 5)/Synthetic macroscopic and microscopic description of the levels observed under the Chemin Saulnier, according to the section 1 (fig. 5)

\begin{tabular}{|c|c|c|c|c|}
\hline $\begin{array}{l}\text { Stratigraphie } \\
\text { sous le } \\
\text { chemin }\end{array}$ & $\begin{array}{l}\text { Observations } \\
\text { macroscopiques }\end{array}$ & $\begin{array}{l}\text { Observations } \\
\text { micromorphologiques }\end{array}$ & Datation & Interprétation \\
\hline \multirow[b]{2}{*}{ Niveau 5} & & & $\begin{array}{l}\text { De l'époque } \\
\text { antique à } \\
\text { aujourd'hui }\end{array}$ & $\begin{array}{l}\text { Superposition } \\
\text { de niveaux de } \\
\text { circulation } \\
\text { alternant états } \\
\text { en terre et états } \\
\text { en pierre. }\end{array}$ \\
\hline & $\begin{array}{l}\text { Niveau limoneux } \\
\text { à limono- } \\
\text { argileux brun- } \\
\text { jaune verdâtre, } \\
\text { compact, } \\
\text { structure } \\
\text { lamellaire } \\
\text { compactée très } \\
\text { développée, } \\
\text { traces } \\
\text { d'oxydations. }\end{array}$ & & $\begin{array}{l}\text { Fin } \mathrm{I}^{\mathrm{er}} \text { siècle } \\
\text { av. J.-C. et } 1 \\
\text { re moitié du } \\
\text { I }^{\text {er }} \text { siècle } \\
\text { apr. J.-C. : } \\
\text { fragment de } \\
\text { vase. }\end{array}$ & $\begin{array}{ll}\text { Niveau de } & \text { de } \\
\text { circulation en } \\
\text { terre très } \\
\text { fréquenté. } \\
\text { Couche } \\
\text { d'accumulation } \\
\text { par apport } \\
\text { sédimentaire } \\
\text { naturel et } \\
\text { anthropique. }\end{array}$ \\
\hline
\end{tabular}




\begin{tabular}{|c|c|c|c|c|}
\hline & $\begin{array}{l}\text { En surface, sur } \\
2 \text { à } 4 \mathrm{~cm} \text { : couche } \\
\text { limono-argileuse } \\
\text { à argileuse, brun- } \\
\text { noir, dur, } \\
\text { structure } \\
\text { lamellaire. }\end{array}$ & $\begin{array}{l}\text { Limons quartzeux, quelques } \\
\text { sables fins à très fins } \\
\text { calcaires. Microstructure } \\
\text { lamellaire à porosité } \\
\text { subhorizontale. Très rares } \\
\text { papules et intercalations } \\
\text { d'argiles hyalines. Rares } \\
\text { pseudomorphoses racinaires } \\
\text { calcifiées et quelques } \\
\text { hyporevêtements } \\
\text { micritiques. } \\
\text { d'hydromorphie. Charbon et } \\
\text { matière organique huméfiée. } \\
\text { Faible bioturbation. }\end{array}$ & $\begin{array}{lr}\text { Entre le } \\
\text { Bronze } \\
\text { ancien et le } \\
\text { début de } \\
\text { l'Âge du } \\
\text { Fer. }\end{array}$ & $\begin{array}{l}\text { Niveau de } \\
\text { circulation } \\
\text { régulièrement } \\
\text { piétiné } \\
\text { (correspond à } \\
\text { l'horizon A du } \\
\text { paléosol). }\end{array}$ \\
\hline Niveau 3 & $\begin{array}{l} \\
\\
\\
\text { Niveau } \\
\text { organique } \\
\text { limono-argileux } \\
\text { brun-jaune } \\
\text { sombre, compact } \\
\text { à dur, structure } \\
\text { polyédrique à } \\
\text { grumeleuse, } \\
\text { fréquents } \\
\text { microcharbons. }\end{array}$ & 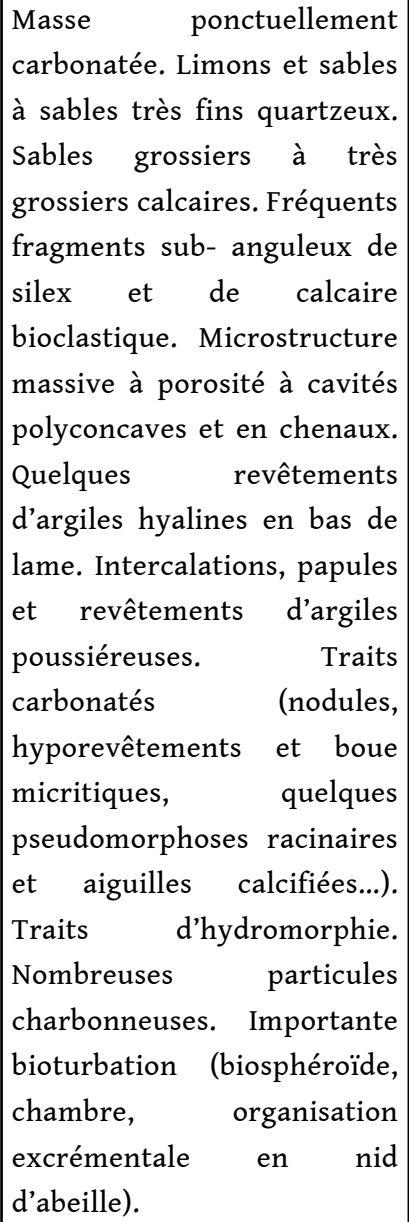 & $\begin{array}{l}\text { Bronze } \\
\text { ancien : } \\
3416 \quad \pm \quad 33 \\
\text { BP, soit } \\
\text { 1776-1625 } \\
\text { av. J.-C. cal. }\end{array}$ & $\begin{array}{l}\text { Paléosol } \\
\text { développé aux } \\
\text { dépens du Bt et } \\
\text { dont la surface } \\
\text { est mise à nue, } \\
\text { peut-être par } \\
\text { brûlis. }\end{array}$ \\
\hline
\end{tabular}




\begin{tabular}{|c|c|c|c|c|}
\hline Niveau 2 & $\begin{array}{l}\text { Limon argileux } \\
\text { brun-rouge } \\
\text { orangé à jaune, } \\
\text { très compact, } \\
\text { structure } \\
\text { prismatique } \\
\text { assez } \\
\text { développée, } \\
\text { microcharbons } \\
\text { épars, quelques } \\
\text { concrétions } \\
\text { carbonatées. }\end{array}$ & 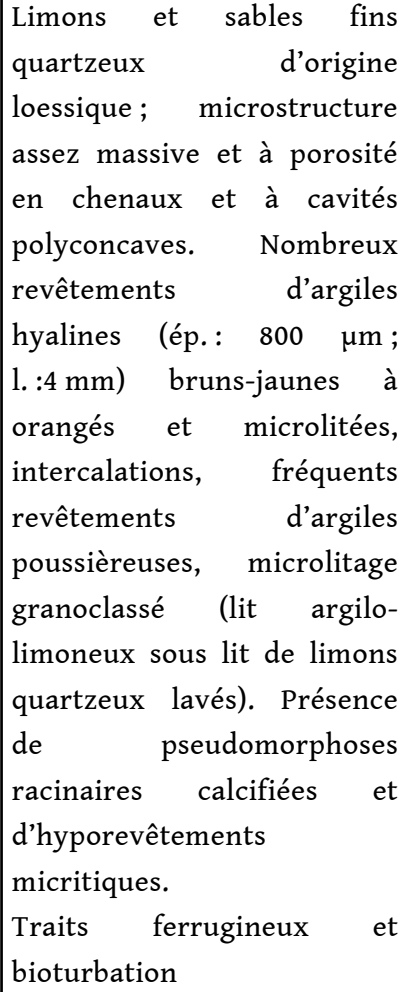 & $\begin{array}{l}\text { Néolithique } \\
\text { final : } 4182 \pm \\
36 \text { BP, soit } \\
2817-2661 \\
\text { av. J-C. cal. }\end{array}$ & $\begin{array}{l}\text { Horizon illuvial } \\
\text { (Bt) issu d'un } \\
\text { luvisol tronqué. }\end{array}$ \\
\hline
\end{tabular}

Photo 2 : Revêtement d'argiles hyalines (Rh) caractéristique d'un horizon illuvial (lame 1 : horizon illuvial fossilisé ; LPNA)/An illuvial clay coating (Rh) characteristic of an illuvial horizon (thin section 1 , illuvial fossilized horizon, LPNA)

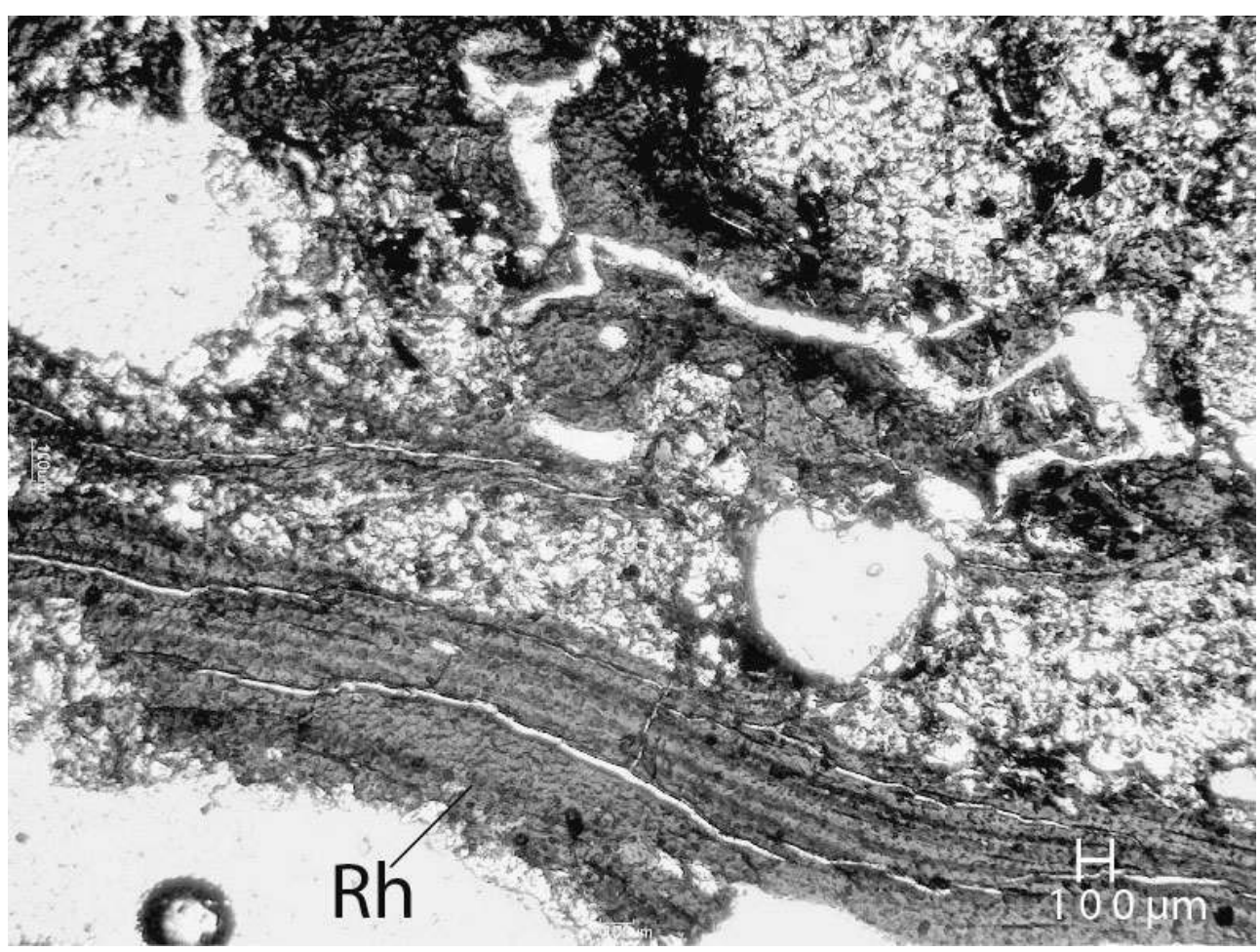


Photo 3 : Papule $(P)$ : fragment de revêtement d'argiles hyalines issu de l'érosion d'un horizon illuvial (lame 1 : horizon illuvial fossilisé ; LPNA)/Papules (P): fragment of illuvial clay coating stemming from the erosion of an illuvial horizon (thin section 1, illuvial fossilized horizon, LPNA)

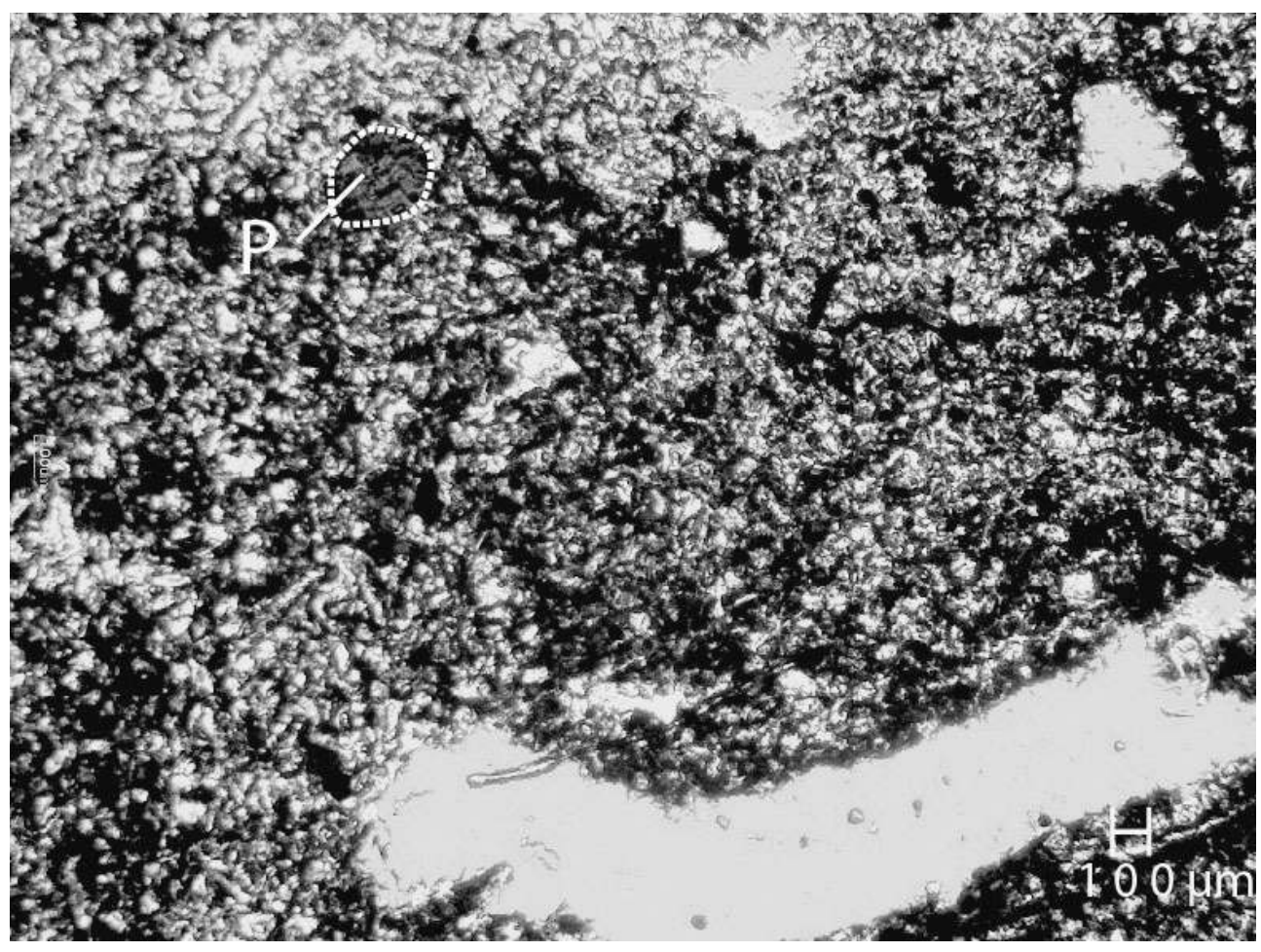

Photo 4 : Accumulation d'argiles poussiéreuses (Rp) attestant d'une ouverture du milieu (lame 16 : horizon illuvial fossilisé ; LPNA)/An accumulation of dusty clay (Rp) providing evidence of a clearing in the environment (thin section 16, illuvial fossilized horizon, LPNA)

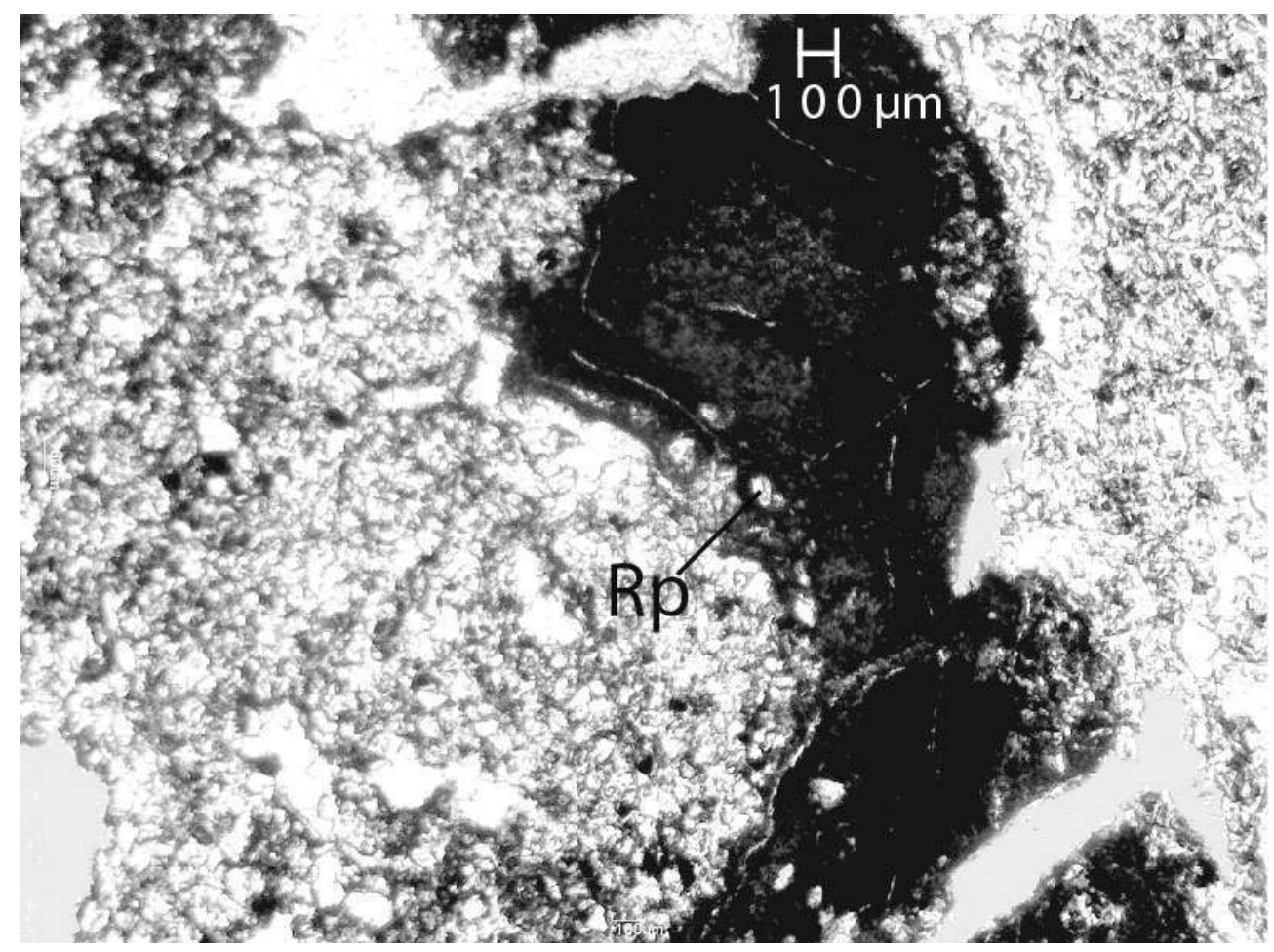


Photo 5 : Croûte de battance $(\mathrm{Cb})$ témoignant d'une mise à nue des sols (lame 34 : Bt situé hors du secteur du Chemin Saulnier ; LPNA)/A structural crust (Cb) indicating bare ground (thin section $34, B t$ situated in the sector of the Iron Age enclosures, LPNA)

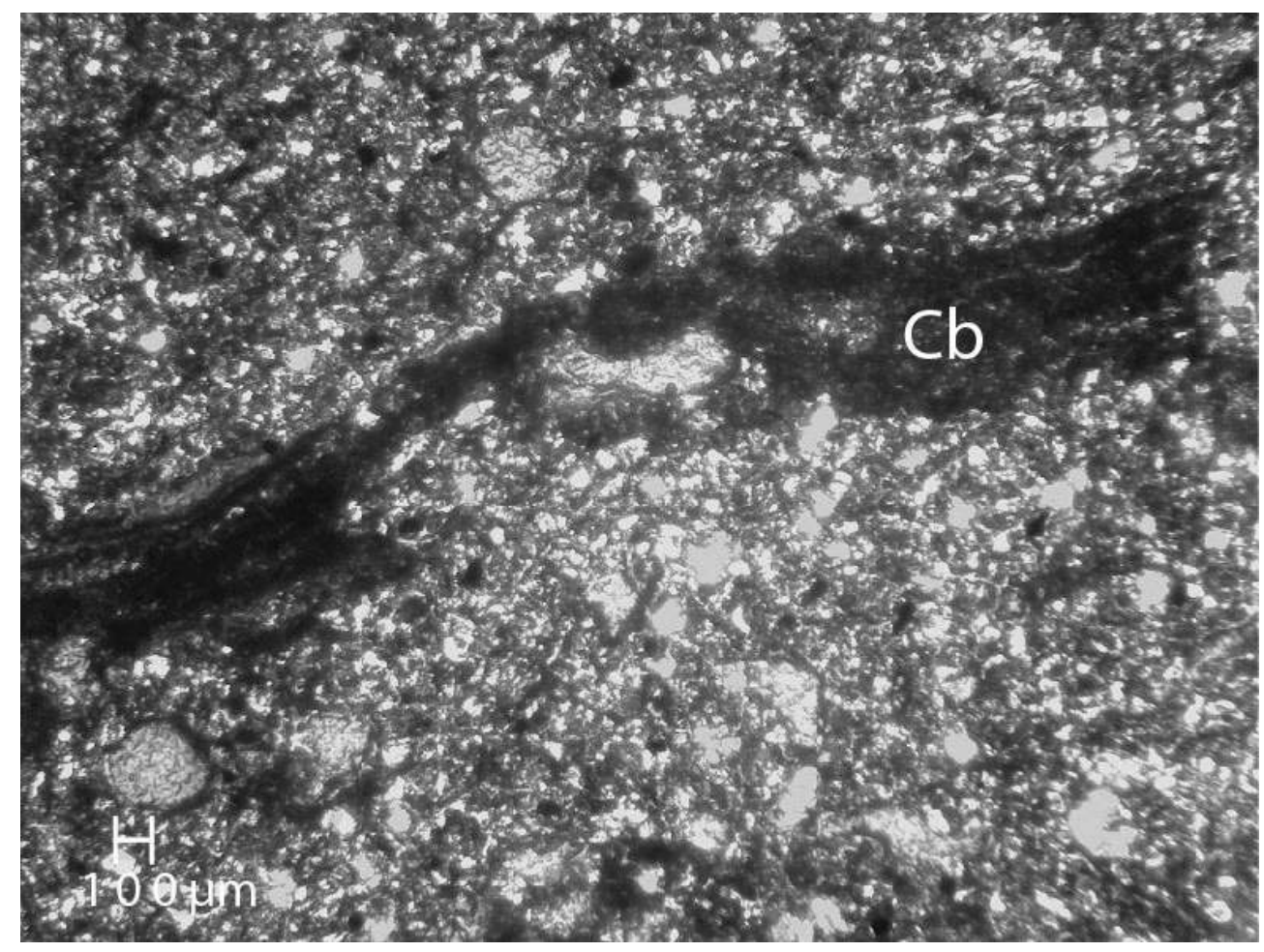

Le niveau 3 : L'horizon limono-argileux brun sombre à brun-noir en surface (tableaux 2 et 4)

L'observation au microscope des lames minces prélevées dans cet horizon révèle dans sa partie inférieure, la présence de revêtements d'argiles hyalines en position de vide. Ceuxci permettent d'identifier une formation développée aux dépens de l'horizon illuvial sousjacent (Fedoroff et Courty, 2002). Les fragments de revêtements d'argiles hyalines (papules) ainsi que les agrégats argilo-limoneux arrondis comportant des grains de calcaires bioclastiques indiquent que ce niveau s'est aussi formé par des apports sédimentaires colluviés résultant de l'érosion de l'horizon illuvial sous-jacent et de sédiments carbonatés. Ce niveau est aussi caractérisé par de nombreux traits d'origine biologique. Il s'agit de carbonatations secondaires (biosphéroïdes calcitiques de lombric, pseudomorphoses racinaires et aiguilles calcifiées) indiquant la présence d'une ancienne couverture végétale (Berger, 2006; Fedoroff et Courty, 2002; Verrechia, 2002). Cette activité biologique se manifeste également au travers de la porosité constituée de chenaux et de cavités polyconcaves. Ces dernières suggèrent l'effondrement d'une structure pédologique originelle qui devait être bien développée (Berger, 2006 ; Fedoroff et Courty, 2002). En résumé, l'ensemble de ces observations associé à la couleur sombre de cet horizon indiquent qu'il s'agit d'un paléosol développé aux dépens de l'horizon illuvial Bt et d'apports colluviaux. Le développement de ce niveau suggère l'existence d'une période de stabilité paysagère postérieure à la troncature du luvisol mais antérieure à sa fossilisation par le Chemin Saulnier. 
21 L'analyse montre, par ailleurs, la présence d'intercalations argileuses et de ponctuations ferro-manganiques indiquant des phases d'engorgements temporaires (Fedoroff et Courty, 1994, 2002). Ces dernières sont d'autant plus prononcées que le paléosol est riche en matière organique (Fechner et al., 2006). On note également la présence de revêtements d'argiles poussiéreuses ainsi que de nombreuses particules charbonneuses et de microcharbons de bois. L'ensemble de ces traits micromorphologiques, associé à l'effondrement de la structure pédologique d'origine, suggère qu'une période de dégradation de ce paléosol a suivi la période de stabilité qui a permis son développement. Les revêtements poussiéreux indiquent sa mise à nu qui pourrait être liée à une action de défrichement par brûlis si l'on considère les nombreux microcharbons observés (Sordoillet, 1999 ; Fedoroff et Courty, 2002 ; Weber-Tièche et Sordoillet, 2008).

22 Enfin, ce paléosol présente, dans sa partie supérieure, une importante structuration lamellaire qui se manifeste par une porosité fissurale horizontale et verticale subparallèle délimitant des agrégats angulaires d'un centimètre de large environ (Courty et al., 1994) (photo 6). Ces traits structuraux très développés se sont créés sous l'effet d'un piétinement intense et confirment que cet horizon constituait la première assise du Chemin Saulnier. Il semble qu'après son défrichement ce paléosol a été très rapidement protégé par l'installation du chemin qui en a assuré ainsi la conservation.

\section{Le niveau 4 : Le niveau limono-graveleux brun (tableau 3)}

Sous le microscope, ce niveau présent en bordure du chemin, contient quelques fragments de revêtements d'argiles hyalines et se caractérise par une fraction grossière constituée majoritairement d'éléments sub-anguleux de calcaires bioclastiques et de silex. Les traits pédologiques observés sont essentiellement dus aux bioturbations. On note, par ailleurs, la présence de nombreux revêtements d'argiles poussiéreuses qui témoignent d'un horizon régulièrement dénudé. Ces caractéristiques micromorphologiques indiquent que ce niveau correspond à une colluvion, issue de l'érosion des paléosols et des niveaux de circulation empierrés, qui s'est accumulée le long du chemin. À partir de la création du chemin, et au fur et à mesure des mises en valeur agricoles, ce rideau de culture s'est formé par érosion des terrains adjacents. Son accrétion rapide a permis la fossilisation du paléosol sous-jacent. 
Photo 6 : Pseudomorphose racinaire en cours de dissolution (Ps) traduisant la présence d'une couverture végétale (lame 2 : paléosol ; LPA)/A pseudomorph of calcified root in the course of dissolution (Ps) translating the presence of a plant cover (thin section 2, paleosol, LPA)

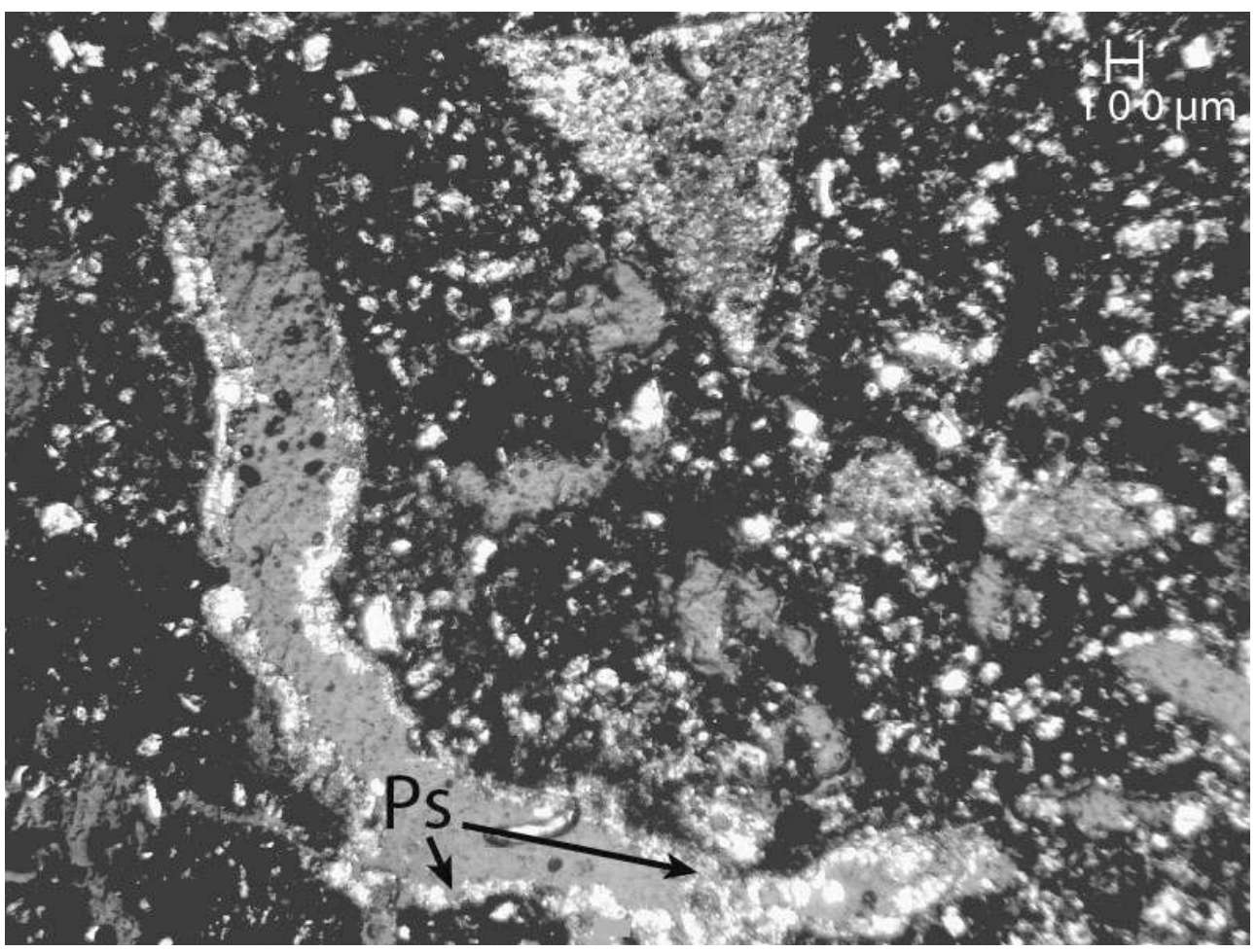

Photo 7 : Microstructure lamellaire formée par l'action mécanique du piétinement (lame 32 : chemin en terre, LPA)/A lamellar microstructure formed by the mechanical action of trampling (thin section 32, road with hard packed surface, LPA)

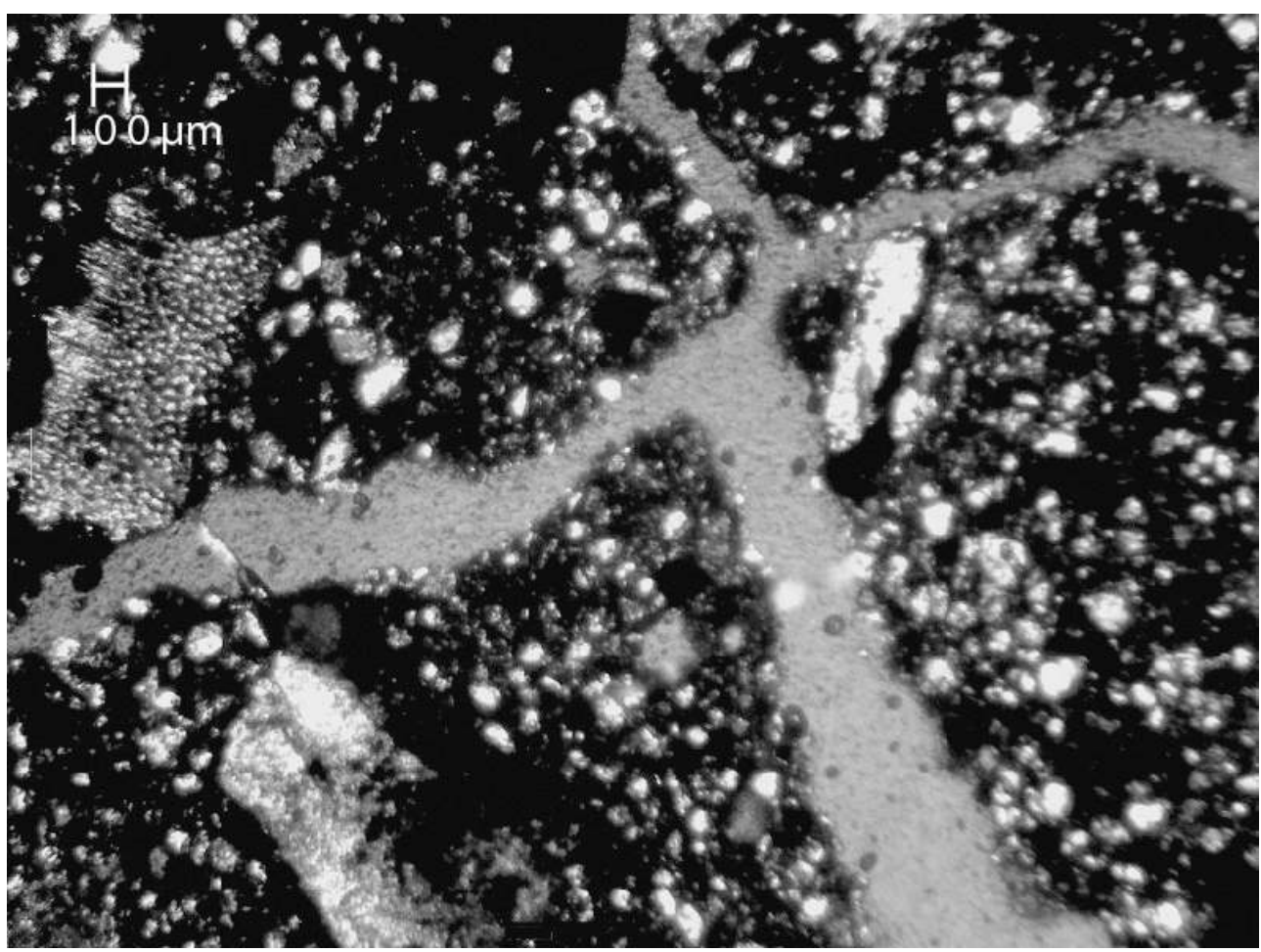




\section{Synthèse de l'étude micromorphologique des pédo-sédiments observés dans la zone de fouille}

La synthèse des résultats micromorphologiques acquis à l'occasion de l'étude de ce chemin ancien permet de dégager plusieurs phases dans l'évolution des sols dans ce secteur de la Plaine de Caen. Après une longue période de stabilité paysagère, favorable au développement d'un luvisol, une première grande phase érosive intervient sur l'ensemble du secteur comme l'atteste la troncature généralisée de ce luvisol jusqu'à son horizon Bt (niveau 2). Cet épisode érosif est suivi d'une période favorable à la reprise de la pédogénèse comme en témoigne le paléosol (niveau 3) qui s'est développé aux dépens de l'horizon illuvial conservé du luvisol. Ce développement indique une interruption dans l'exploitation des sols qui a entraîné la reconquête d'un couvert végétal protecteur.

Sous le chemin et les rideaux de cultures associés qui, dans un troisième temps, viennent le couvrir (niveau 4), ce paléosol contient en surface de nombreux microcharbons qui pourraient résulter d'un défrichement par brûlis. Il n'est toutefois pas possible de savoir si ce dernier est directement lié à l'installation du chemin ou s'il était à l'origine plus étendu. Quoiqu'il en soit, le chemin a manifestement été mis en place très peu de temps après la mise à nu du paléosol comme le montrent à son sommet des indices importants de tassement. Ce premier niveau de circulation est, par la suite, recouvert par la succession de quatre autres états, dont le chemin actuel constitue l'ultime développement (niveau 5). Les observations micromorphologiques qui ont été effectuées dans les différents états en terre attestent une circulation continue et importante qui se traduit par une structure lamellaire très développée dans ces niveaux et par l'absence de trait biologique.

\section{Restitution de l'histoire du paysage holocène à l'échelle du site de Banneville-la-Campagne}

Dans le cadre de la présente étude, deux phases de biostasie entrecoupées de deux phases de rhexistasie, ont été mises en évidence pour la période holocène dans ce secteur de la Plaine de Caen. Le calage chronologique de ces événements pédo-sédimentaires a été établi par l'intermédiaire des rapports stratigraphiques entre les horizons considérés et les vestiges d'occupation rencontrés ou par le recours à des datations radiométriques. Les observations ainsi effectuées permettent dès lors de proposer une restitution des différentes étapes de l'histoire du paysage holocène et de mesurer l'impact anthropique sur son évolution (fig. 7).

La première phase de stabilité (étape 1) correspond au développement d'un luvisol, à compter du début de l'Holocène (Camuzard, 2000 ; Besnard-Vauterin et al., 2006). Ce type de sol, communément rencontré en Europe tempérée, est significatif à la fois d'un climat à précipitations relativement régulières, dans lequel alternent saisons humides et saisons sèches, et de la présence d'un couvert végétal continu permettant une bonne protection de la surface du sol (Duchaufour, 1983 ; Fedoroff et Courty, 1994, 2002).

Intervient ensuite une première phase érosive (étape 2), attestée par la troncature de ce même luvisol observée sur l'ensemble de la zone étudiée. Elle se manifeste par l'absence des horizons superficiels A et $\mathrm{E}$ au-dessus du Bt. Cette troncature peut être considérée 
comme le résultat d'une exploitation des sols qui aurait débutée au cours du Néolithique final. En effet, une datation radiométrique réalisée sur des microcharbons issus de ce Bt fossilisé par le Chemin Saulnier, situe cet épisode érosif dans le dernier tiers du Néolithique final (2 817-2 661 av. J.-C. cal.). Une telle datation s'accorde avec la présence aux voisinages immédiats de la zone étudiée de traces d'établissements néolithiques détectées lors de campagnes de prospection de surface (Jasmin, 1942-1945) qui témoignent, elles aussi, d'une fréquentation intense de cette zone au cours du Néolithique.

Une nouvelle phase de stabilité du paysage (étape 3) succède à cette phase de dégradation des sols. Elle est attestée par le développement du paléosol aux dépens du Bt tronqué. Cette reprise de la couverture végétale correspondrait à une période de non-exploitation des sols à des fins agricoles, qui se situerait entre la fin du Néolithique final et le Bronze ancien. En effet, à cette période, le paléosol subit une nouvelle mise en culture. Le calage de cette deuxième phase d'anthropisation du paysage à l'ultime fin du Néolithique ou au cours du Bronze ancien (1776-1625 av. J.-C. cal.) a été défini à partir d'une datation radiométrique réalisée sur des microcharbons prélevés dans le paléosol (étape 4). La disparition de ce paléosol en dehors de l'emprise au sol du chemin et des rideaux de culture accumulés le long de son tracé ne permet pas de trancher entre l'hypothèse d'un défrichement ponctuel destiné à installer le chemin et celle d'une ouverture plus large du paysage.

Quoiqu'il en soit, ce n'est qu'à compter de la transition Premier - Second Âge du Fer que l'activité agricole semble s'intensifier comme l'indique l'implantation des enclos fossoyés, qui correspond à trois phases d'occupation se succédant au cours de l'Âge du Fer. Des datations radiométriques, réalisées sur les restes osseux prélevés dans deux sépultures associées aux enclos les plus anciens, fixent leur installation vers la fin du Premier Âge du Fer ou au début du Second (595-403 av. J.-C. cal.) (tableau 1). Le mobilier déposé dans la tombe la plus récente atteste l'usage de ces enclos au début de La Tène.

31 La présence de ces établissements doit avoir entraîné la mise en culture des terres, déclenchant ainsi l'érosion du paléosol en dehors de l'endroit où il a été préservé par l'existence d'un chemin en activité (étape 5). Le chemin en terre battue qui s'implante directement sur le paléosol défriché connaît ensuite une fréquentation dense pendant au moins un demi-millénaire, matérialisée par l'épais niveau de circulation en terre qui s'est formé par accumulation au-dessus du paléosol piétiné.

Par la suite, la chaussée sera aménagée alternativement en pierre et en terre battue (étape 6). L'ancienneté de cet axe de circulation est dès lors avérée et son maintien à un même emplacement depuis le début de l'Âge du Fer montre son rôle structurant dans le paysage.

\section{De l'intra-site à l'extra-site}

La présente étude révèle que, pour la zone fouillée, la troncature du luvisol semble intervenir au Néolithique. Cette phase précoce d'érosion apparait cependant comme un phénomène localisé puisque ces défrichements n'ont pas eu une ampleur suffisante pour permettre le transfert des particules de sols arrachées sur le plateau vers les petites vallées avoisinantes. En effet, cette phase érosive néolithique n'a eu aucune répercussion dans l'évolution morpho-sédimentaire du vallon du ruisseau de Cagny localisé à un 
kilomètre en direction du sud et qui a fait l'objet d'études paléoenvironnementales récentes (Germain-Vallée et Lespez, ce volume) (fig. 1).

Après cette première phase érosive, une période de stabilité est suggérée par le développement d'un second paléosol. De telles pédogenèses postérieures au Néolithique ont été révélées par les analyses micromorphologiques réalisées récemment sur les sédiments de comblement de structures fouillées à Tilly-la-Campagne et Fontenay-leMarmion (Germain-Vallée et Lespez, ce volume). Toutefois dans notre zone d'étude, cet épisode de déprise n'a pas permis le développement d'un luvisol post-néolithique, comme cela a été observé sur les autres sites, mais s'est soldé par la formation d'un paléosol constitué d'un unique horizon organique. Cette dernière particularité pourrait s'expliquer par la durée limitée, d'environ un millénaire, de cette déprise. En effet, la datation obtenue indique que la fin de cette période de stabilité se situe à la fin du Néolithique ou dans le courant du Bronze ancien. Comme pour celle attribuée au Néolithique, cette deuxième phase d'anthropisation n'a pas eu de répercussion dans la petite vallée proche du ruisseau de Cagny (Germain-Vallée et Lespez, ce volume). Cette constatation, couplée à l'absence de vestiges contemporains dans la zone fouillée, suggère que l'ouverture du milieu résulte d'un événement localisé et de faible ampleur. En revanche, si l'anthropisation du paysage reste apparemment discrète à l'Âge du Bronze, elle s'intensifie nettement à l'Âge du Fer avec l'implantation, dans la zone étudiée, d'enclos à vocation agro-pastorale. Cette densification de l'occupation, observée dans la zone fouillée, concorde avec les observations géomorphologiques effectuées dans la vallée du ruisseau de Cagny : un colmatage par des limons de débordement de ce vallon est en effet intervenu, comme dans la plupart des vallées bas-normandes, à partir du début de l'Âge du Fer (Germain-Vallée et Lespez, 2006 ; Lespez et Germain-Vallée, 2010 ; GermainVallée et Lespez, ce volume). Elle s'accorde également avec les études archéologiques portant sur les plateaux de la rive droite de la basse vallée de l'Orne, à quelques kilomètres à l'ouest du secteur étudié. Ces études ont révélé une forte structuration du paysage tout au long de l'Âge du Fer, attestée par un système parcellaire dense dans lequel s'insèrent de nombreuses unités agricoles reliées entre elles par des chemins (Le Goff, 2000-2002 ; Besnard-Vauterin, 2009).

Figure 7 : Reconstitution de l'évolution des formations pédo-sédimentaires fossilisées par le Chemin Saulnier à Banneville-la-Campagne/Cagny./Reconstruction of the evolution of the pedosedimentary formations fossilized by the Chemin Saulnier at Banneville-la-Campagne/Cagny

\section{Conclusion}

La présence du Chemin Saulnier dès l'Âge du Bronze a préservé le second paléosol qui, partout ailleurs, a très certainement été détruit par l'intensification, au cours de l'Âge du Fer, des travaux de mise en valeur agricole des terrains avoisinants. Une telle datation précoce de chemins de la Plaine de Caen a déjà été envisagée en se fondant sur la corrélation qui semble exister entre la dispersion des enclos funéraires de l'Âge du Bronze et des axes de circulations les plus importants empruntés par les hommes et les produits depuis l'Âge du Fer jusqu'à la période Moderne (Chevalier, 2009; Desloges, à paraître). L'étude réalisée en 2009 sur un petit tronçon du Chemin Saulnier s'accorde avec cette hypothèse, même si cet axe n'avait pas été retenu par les auteurs de la recherche précédemment évoquée. Elle atteste une nouvelle fois de l'importance de la période protohistorique dans la mise en place d'un réseau viaire que les arpenteurs gallo-romains 
n'ont fait que réaménager en substituant aux anciens chemins de terre des voies plus ou moins densément empierrées (fig. 1) (Robert, 2009). L'utilité de ces cheminements et leur fonction dans la structuration des paysages, justifie leur maintien en activité sur la très longue durée, de l'Âge du Bronze à nos jours.

Cette étude a, par ailleurs, montré l'intérêt de combiner analyses géomorphologiques, micromorphologiques et archéologiques dans le cadre d'une étude d'un chemin ancien. Cette approche a permis d'analyser des formations pédo-sédimentaires anciennes fossilisées par ce chemin et de reconstituer ainsi l'évolution du paysage à l'échelle du site. La confrontation de ces résultats à ceux obtenus ailleurs dans la Plaine de Caen a permis de dégager des épisodes de stabilité et de dégradation du paysage, de portée locale mais participant aussi à des phénomènes qui s'observent plus largement dans la Plaine de Caen. Reste à connaître la valeur de ces observations faites sur un court tronçon du Chemin Saulnier, en les confrontant à des études similaires réalisées en d'autres points du parcours de ce chemin et sur d'autres axes de cheminement considérés comme anciens qui sillonnent la Plaine de Caen.

\section{BIBLIOGRAPHIE}

BERGER J.-F., 2006. Apports récents de la paléopédologie à la définition des phases de stabilités des pédopaysages holocènes nord-méditerranéens, dans ALLÉE P. et LESPEZ L. (eds.), L'érosion entre société, climat et paléoenvironnement, Clermont-Ferrand, colloque Nature et société 3, Presses universitaires Blaise-Pascal, p. 47-62.

BESNARD-VAUTERIN C.-C., CHANCEREL A., MARCIGNY C., 2006. L'enceinte de l'Âge du Bronze moyen de l'Étoile (Mondeville), dans CHANCEREL A., MARCIGNY C., GHESQUiÈre E. (dir.), Le plateau de Mondeville (Calvados), du Néolithique à l'Âge du Bronze, Paris, Édition de la Maison des Sciences de l'Homme, Documents d'archéologie française 99, p. 114-116.

BESNARD-VAUTERIN C.-C. (dir.), 2009. En Plaine de Caen, une campagne gauloise et antique, L'occupation du site de l'Étoile à Mondeville, Rennes, PUR, coll. « Archéologie \& Culture », 312 p.

BERTIN D., 1975. Introduction à une étude de l'époque gallo-romaine en Basse-Normandie : carte de répartition des voies et des sites gallo-romains de Basse-Normandie, Annales de Normandie, p. 67-74.

BULLOCK P., FEDOROFF N., JONGERIUS A., STOOPS G., TURSINA T., BABEL U., 1985. Handbook for soil thin section description, Wolverhampton, Waine Research Publications, $152 \mathrm{p}$.

CAMUZARD J.-P., 2000. Les sols marqueurs de la dynamique des systèmes géomorphologiques continentaux, Thèse de $3^{\mathrm{e}}$ cycle, Caen, $509 \mathrm{p}$.

CARPENTIER V., GHESQUIÈRE E., MARCIGNY C. (dir.), 2006. Grains de sel, sel et salines du littoral bas-normand (préhistoire - XIX ${ }^{\mathrm{e}}$ siècle), Entre Histoire et Archéologie, Saint-Malo/Rennes, AMARAI/CeRAA, Les Dossiers du CeRAA, Suppl., nº AC, 182 p.

CHEVALIER A., 2009. Espaces funéraires et réseaux viaires protohistoriques en Basse-Normandie, deux approches mathématiques de l'espace, Les nouvelles de l'archéologie, $\mathrm{n}^{\circ}$ 115, mars, p. 44-51. 
COURTY M. A., FEDOROFF N., 2002. Micromorphologie des sédiments archéologiques, dans Misкovsкy J.-C. (ed.), Géologie de la Préhistoire : méthodes, techniques et applications, Paris/Perpignan, Editions GEOPRE/Presses Universitaires de Perpignan, p. 277-316.

COURTY M. A., GOLDBERg P., MACPHAIL R.I., 1989. Soils and Micromorphology in Archaeology, Cambridge manuals in archaeology, Cambridge University Press, 344 p.

COURTY M. A., GOLBERG P., MACPHAIL R. I., 1994. Ancient people - Lifestyles and cultural patterns, dans WILDING L. and OLESSHKO K (eds.), Micromorphological indicators of anthropogenic effects on soils. $15^{\text {th }}$ World congress of Soil Science, Mexico, vol 6a, p. 250-269.

COUTARD J.-P., HELLUIN M., LAUTRIDOU J.-P., OZOUf J.-C., PELLERIN J., 1970. Les loess de la Campagne de Caen, Bulletin du Centre de Géomorphologie de Caen, $\mathrm{n}^{\circ}$ 8, p. 1-30.

DELACAMPAGNE F., DESLOGES J., GIGOT P., SAN JUAN G., 1992, Vol à travers les âges. La prospection archéologique aérienne dans le Calvado, Caen, Conseil Général du Calvados, 107 p.

DESLOGES J., 2001. Les prospections aériennes dans les plaines de grande culture du Calvados et de l'Orne, Bilan scientifique régional, SRA Basse-Normandie, p. 98-99.

-, 2002. Les prospections aériennes dans les plaines de grande culture du Calvados et de l'Orne, Bilan scientifique régional, SRA Basse-Normandie, p. 108-109.

-, (à paraître). Le plateau littoral de la Plaine de Caen, un cas de topographie historique, dans CARPENTIER V., MARCIGNY C. (dir.), Actes de la Table Ronde " Des hommes aux champs ", pour une archéologie des espaces ruraux dans le nord de la France, du Néolithique au Moyen Âge, octobre 2008, p. 53.

DESLOGES J., GIGOT P., AUGER N., 2003. Les prospections aériennes dans les plaines de grande culture du Calvados et de l'Orne, SRA Basse-Normandie, Bilan scientifique régional, p. 121.

DESLOGES J., GIGOT P., ROPARS A., AUGER N., 2004. Les prospections aériennes dans les plaines de grande culture du Calvados et de l'Orne, SRA Basse-Normandie, Bilan scientifique régional, p. 12-123.

DORANLO R., 1936-1937. Notes d'archéologie antique : les voies de la « civitas » des Lexovii, Bulletin de la Société normande d'études préhistoriques, $\mathrm{n}^{\circ} \mathrm{XXIX}$, p. 145-200.

Duchaufour P., 1983. Pédologie, 1. Pédogenèse et classification, $2^{\mathrm{e}}$ édition, Paris, Edition Masson, 491 p.

DUVERNOIS B., 1987. Un habitat rural gallo-romain à Bréville-les-Monts (Calvados), Rapport de fouille de sauvetage, SRA Basse-Normandie.

FECHNER K., BOSQUET D., BLANCQUAERT G., 2006. Indices pédologiques liés au (re)creusement et à l'utilisation des fosses : les cas exemplaires de Remicourt (Néolithique ancien, Wallonie, Belgique) et Dourges (La Tène, Pas-de-Calais, France), dans FRÈrE-SAUTOT M.-C. (dir.), Des trous ... Structures en creux pré- et protohistoriques, Actes du colloque de Dijon et Baume-les-Messieurs, 24-26 mars 2006, p. 47-73.

FEDOROFF N., COURTY M-A, 1994. Organisation du sol aux échelles microscopiques, dans Duchaufour P. et SOUCHIER B. (dir.), Pédologie 2. Constituants et propriétés du sol, deuxième édition, Paris, Masson, p. 349-375.

FEDOROFF N., COURTY M.-A., 2002. Paléosols et sols reliques, dans MISKOvSKY J.-C. (dir.), Géologie de la Préhistoire : méthodes, techniques et applications, Paris/Perpignan, éditions GEOPRE/Presses Universitaires de Perpignan, p. 277-316.

FLOTTÉ D., 2005. Bréville-les-Monts, Bilan Scientifique Régional de Basse-Normandie, p. 39-41. 
GEBHARDT A., 1990. Evolution du paléopaysage agricole dans le nord-ouest de la France. Apport de la micromorphologie, Thèse de doctorat, Université de Rennes I, 175 p.

GERMAIN-VALLÉE C., LESPEZ L., 2006. Dynamique holocène d'un fond de vallée normand (vallée du Laizon, Calvados). Approche géomorphologique et micromorphologique, dans ALLÉE P. et LESPEZ L. (dir.), L'érosion entre société, climat et paléoenvironnement, Clermont-Ferrand, colloque Nature et société 3, Presses universitaires Blaise-pascal, p. 279-284.

,- 2011. L'apport de recherches géomorphologiques et micromorphologiques récentes à l'archéologie des paysages de la Plaine de Caen (Calvados, Basse-Normandie), Norois, ce volume, p. $143-178$.

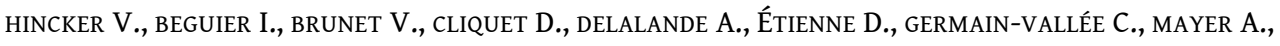
SAVARY X., 2009. Contournement sud-est de Caen. Barreau de raccordement A13 - RN 13, rapport final de fouille, Caen, Service d'Archéologie du Conseil Général du Calvados, SRA Basse-Normandie, 3 vol., $185 \mathrm{p}$.

JASMIN P., 1942-1945. Note sur ses découvertes archéologiques à Banneville-la Campagne (Calvados, canton de Troarn), Bulletin de la société des antiquaires de Normandie, tome XLIX, p. 427-428.

LE GOFF E. (dir.), 2000-2002. Les occupations protohistoriques et antiques de la ZAC «Object'Ifs Sud », Ifs (Calvados), Document Final de Sauvetage urgent, 4 volumes.

LESPEZ L., GERMAIN C., 2010. Les paléoenvironnements de l'Âge du Fer en Basse-Normandie : Etat des connaissances et problèmes posés, dans BARRAL P., DELRIEU F., LE GOFF, E., MARION S., VILLARD LE TIEC A. (dir.) Actes du XXIII colloque International de l'AFEAF, Besançon, Presses Universitaires de Franche-Comté ; Annales littéraires, série Environnement, Société et Archéologie, p. 35-49.

MUSSET L., 1983. Sur les chemins sauniers de la Normandie médiévale, Annales de Normandie, fasc. $\mathrm{n}^{\circ}$ 2, juin, p. 177. (Arch. Dép. Calvados, H 6510).

ROBERT S., 2009. L'héritage pré et post-romain dans les réseaux routiers anciens, Les nouvelles de l'archéologie, n²115, mars 2009, p. 23-30.

SORDOILLET D., 1999. Géoarchéologie des sites préhistoriques Holocènes, Formation doctorale PAL\&SED, Université de Bourgogne, Centre des sciences de la Terre, $297 \mathrm{p}$.

VAN VLIET-LANÖ̈ B., 1988, Le rôle de la glace de ségrégation dans les formations superficielles de l'Europe de l'Ouest. Processus et héritages, Thèse d'État Paris I., 854 p.

VERRECHIA P.-E., 2002. Géodynamique du carbonate de calcium à la surface des continents, dans MISKovsKy J.-C. (dir.), Géologie de la Préhistoire : méthodes, techniques et applications, Paris, éditions GEOPRE, Presses Universitaires de Perpignan, p. 233-258.

WEBER-TIECHE I., SORDOILLET D., 2008. Plateau de Bevaix, 4. Étude géologique en contexte archéologique. Office et musée cantonal d'archéologie (Archéologie neuchâteloise numérique, 3), Neuchâtel, p. 126.

\section{RÉSUMÉS}

Cette étude menée dans la Plaine de Caen vise à préciser la genèse et le mode d'évolution d'un chemin ancien appelé Chemin Saulnier. Parallèlement, elle a permis d'étudier des formations pédologiques anciennes fossilisées par ce chemin. Ces formations se caractérisent par un horizon illuvial tronqué, aux dépens duquel s'est développé un paléosol qui, au-delà du chemin qui l'a 
préservé, a été entièrement érodé ou remanié par l'intensification des pratiques agricoles. L'analyse géomorphologique et micromorphologique a ainsi permis de reconstituer à l'échelle intra-site une histoire des paysages de la Plaine de Caen qui montre deux périodes de stabilité, dont l'une est matérialisée par le paléosol, situées entre deux épisodes érosifs d'origine anthropique, datés respectivement du Néolithique final et de la fin du Premier Âge du Fer. Cette étude, couplée aux données archéologiques, a aussi permis d'attester l'existence d'un premier état en terre battue du Chemin Saulnier à l'Âge du Bronze.

This study focusing on the Plain of Caen aims at specifying the genesis and the mode of evolution of an ancient road called the "Chemin Saulnier" (the Saltmaker's Track). At the same time, the project has enabled us to study the pedological formations fossilized by the road. These formations are characterized by an illuvial truncated horizon at the expense of which developed a palaeosol. Beyond the road which protected it, this palaeosol was completely affected or reworked by the intensification of agricultural practices. The geomorphological and micromorphological analyses thus allow us to reconstitute, at the scale of the site itself, the story of the Plain of Caen landscape revealing two periods of stability - auspicious to the development of the paleosol - situated between two anthropogenic phases of erosion, respectively dated to the Final Neolithic and the end of the Hallstatt period (Iron Age). This study, coupled with the archaeological data, also yielded evidence of the existence of an earlier hard-packed earth surface of the "Chemin Saulnier" dating back to the Bronze Age.

\section{INDEX}

Index géographique : Caen (plaine de)

Mots-clés : géoarchéologie, micromorphologie, paléosol, pédologie, phase d'érosion, phase de stabilité

Keywords : micromorphology, geoarchaeology, pedology, paleosol, phase of erosion, phase of stability

\section{AUTEURS}

\section{IRÈNE BÉGUIER}

Service d'Archéologie du conseil général du Calvados 36, rue Fred-Scamaroni - 14000 Caen, France irenebeguier@gmail.com

\section{CÉCILE GERMAIN-VALLÉE}

Service d'Archéologie du conseil général du Calvados 36, rue Fred-Scamaroni - 14000 Caen, France cecile.germain@calvados.fr

\section{VINCENT HINCKER}

Service d'Archéologie du conseil général du Calvados 36, rue Fred-Scamaroni - 14000 Caen, France, vincent.hincker@calvados.fr 